\title{
Pectin remodeling belongs to a homeostatic system and triggers transcriptomic and hormonal modulations
}

François Jobert ${ }^{\mathrm{a}, \mathrm{b} 1}$, Stéphanie Guénin ${ }^{\mathrm{a} \#}$, Aline Voxeur ${ }^{\mathrm{b}, \mathrm{c}}$, Kieran J. D. Lee ${ }^{\mathrm{d}}$, Sophie Bouton $^{b}$, Fabien Sénéchal ${ }^{b}$, Ludivine Hocq $^{b}$, Gaëlle Mongelarda, Hervé Demaillya, Petra Amakorováe, Miroslav Strnad ${ }^{\mathrm{e}}$, Samantha Vernhettes ${ }^{c}$, Gregory Mouille ${ }^{c}$, Serge Pilardf, J. Paul Knox ${ }^{d}$, Ondřej Nováke ${ }^{\mathrm{e}}$, Jérôme Pelloux ${ }^{\mathrm{f}}$, Laurent Gutierrez ${ }^{\mathrm{a}}$

a, CRRBM, b, UMR INRAE 1158 BioEcoAgro Biologie des Plantes et Innovation and f, Plateforme Analytique, Université de Picardie Jules Verne, 80000 Amiens, France.

c, Institut Jean-Pierre Bourgin, Université Paris-Saclay, INRAE, AgroParisTech, 78000, Versailles, France.

d, Faculty of Biological Sciences, University of Leeds, Leeds LS2 9JT Leeds, United Kingdom.

e, Laboratory of Growth Regulators, Faculty of Science, Palacký University and Institute of Experimental Botany, The Czech Academy of Sciences, 78371 Olomouc, Czech Republic.

1 present address: Umeå Plant Science Centre, Swedish University of Agricultural Sciences, 90736 Umeå, Sweden.

\# these authors contributed equally to this work

§ correspondence: laurent.gutierrez@u-picardie.fr ; jerome.pelloux@u-picardie.fr 


\section{ABSTRACT}

Pectins occur in primary cell walls and consist of multiblock co-polymers among which homogalacturonan ( $\mathrm{HG}$ ) is the simplest and most abundant form. Methylesterification patterns of HG are tuned by pectin methylesterases (PMEs), the activities of which are controlled by specific inhibitors (PMEls). By impacting cell wall mechanical properties, PME-mediated regulation of $\mathrm{HG}$ methylesterification plays a major role in several developmental processes, including seed germination and dark-grown hypocotyl elongation. Arabidopsis PME36 is preferentially expressed during the late stage of seed development and, using the knock-out mutant pme36-1, we show here that PME36 is required to implement the characteristic pattern of de-methylesterified pectin in the mature seed. Surprisingly, while this pattern is strongly impaired in pme36-1 mature seed, no phenotypical effect is observed in the mutant during seed germination and dark-grown hypocotyl elongation, suggesting the existence of a compensatory mechanism overcoming the defect in pectin de-methylesterification. By analyzing hormone contents and gene expression, a strong, dynamic, and long-lasting physiological disorder is revealed in the mutant. These results suggest the existence of complex connections between pectin remodeling, transcriptomic regulations and hormonal homeostasis, modulating several physiological parameters to ensure the maintenance of a normal seed-to-seedling developmental program in pme36-1. Considered for a long time as an end-point passive effector mainly involved in modification of cell wall mechanics, the role of pectin methylesterification needs to be reconsidered as a modulator acting upstream of diverse regulatory pathways involved in plant development. 


\section{INTRODUCTION}

Plant cells are confined by semi-rigid walls consisting of a polysaccharide network, composed of cellulose, hemicellulose and pectins, which contains proteins and ions (Chebli et al., 2021). How cell wall plasticity is controlled to regulate plant development and growth is one of the outstanding questions in plant biology (Cosgrove, 2018). Turgor pressure constitutes the driving force for cell expansion and, by fine-tuned spatiotemporal modulations of wall stiffness, plant cells transform this isotropic force into anisotropic growth shaping plant structure (Sampathkumar, 2020). Stiffness modulations imply cell wall remodeling and research to understand the underlying physical mechanisms and molecular regulations is actively debated, as seen for the role of pectins in pavement cell morphogenesis (Cosgrove and Anderson, 2020). During the last decade, pectin has emerged as an important player in morphogenesis regulation, through its effects on wall stiffness modulation that is required for many developmental processes, including seed development and germination (Müller et al., 2013; Levesque-Tremblay et al., 2015a; Scheler et al., 2015), hypocotyl elongation (Pelletier et al., 2010; Peaucelle et al., 2015; Daher et al., 2018), apical hook development (Jonsson et al., 2021), organ emergence (Peaucelle et al., 2008, 2011a, 2011b), leaf epidermis morphogenesis (Majda et al., 2017; Altartouri et al., 2019; Bidhendi et al., 2019; Haas et al., 2020) and asymmetric leaf development (Qi et al., 2017). Pectins, mainly composed of homogalacturonan (HG), are secreted into the wall in a methylesterified form - i.e. pectins with a high degree of methylesterification (DM) - and are then de-methylesterified in muro by the action of pectin methylesterase (PME) proteins, the activities of which are regulated by pectin methylesterase inhibitor (PMEI) proteins. For a long time, pectin de-methylesterification was considered to lead to only to cell wall stiffening, though the production of rigid "egg-box" structures resulting from calcium-mediated cross-linking of HG with low DM (Willats et al., 2001). Conversely, in recent years pectin de-methylesterification has been shown to result in cell wall softening, by a mechanism that remains elusive and in which pectin degradation enzymes - active on low DM pectins - were thought to play a role (Levesque-Tremblay et al., 2015b). Since then, the effect of pectin DM appears to be mostly dependent on the cellular context. In addition, recent insights into the complexity of pectin interactions with other cell wall components (Du et al., 2020), the existence of cell wall domains harboring spatially restricted pectin modifications (Francoz et al., 
2019), and the proposition that pectin nanofilaments are involved in cell expansion (Haas et al., 2020), raise challenges to a full comprehension of the role of pectin methylesterification in cell wall plasticity during plant development.

Deciphering the control of pectin de-methylesterification appears even trickier. PME and PMEI proteins are encoded by multigenic families, composed of 67 and 76 genes in Arabidopsis, respectively (Wang et al., 2013; Hocq et al., 2017a). Efficiency of PMEI inhibitory activity depends on PME/PMEI pairings in a pH-depend manner (Hocq et al., 2017a; Sénéchal et al., 2017). Obviously, the high number of possible combinations restricts the possibility of in planta functional analysis of PME/PMEI interactions. Pectic HG de-methylesterification reactions drive acidification of the cell wall, whereas PME activity optima are at alkaline $\mathrm{pH}$ and inhibitory activity of $\mathrm{PMEl}$ is promoted at acid $\mathrm{pH}$ (Sénéchal et al., 2015; Hocq et al., 2017a; Sénéchal et al., 2017). Thus, pectin demethylesterification activity turns out to be dynamically regulated within cell walls, through feedback loops involving local pH changes (Hocq et al., 2017b), which adds a further level of complexity in the analysis of this enzymatic system.

Knockout mutations in PME genes have been reported to result in developmental defects in only a very limited number of studies. This includes in pollen (Francis et al., 2006; Tian et al., 2006; Leroux et al., 2015), in seeds (Levesque-Tremblay et al., 2015a; Turbant et al., 2016), in stem support (Hongo et al., 2012), during organ emergence (Peaucelle et al., 2011b) and in adventitious rooting (Guénin et al., 2011). Usually, the absence of a phenotype observed in a pme mutant is attributed to gene functional redundancy, although compensatory mechanisms between PME genes have never been extensively characterized. Most of the time, the focus is on the impact of overall pectin methylesterification modification, using plants overexpressing a $P M E$ or - more often - PMEl gene which triggers a steady dominant change in PME activity. Although too drastic to allow the analysis of the fine-tuned control of pectin demethylesterification, such approaches seem appropriate to study the role of pectin methylesterification from a mechanical perspective. Nevertheless, beyond their structural role, pectins and pectin-derived degradation products, such as oligogalacturonides (OGs), interact with wall sensors and are particularly prone to trigger signaling pathways involved in the cell wall integrity (CWI) maintenance (Wolf, 2017; Vaahtera et al., 2019). On the one hand, pleiotropic effects of PME/PMEl gene overexpression brings about loss of pectin remodeling dynamics and overrides the subtle effects it could produce, especially regarding this signaling function. On the 
other hand, absent or subtle phenotypes of pme mutants have never really encouraged in-depth investigations of possible modulations of their physiological parameters. Consequently, our knowledge of the physiological effects resulting from transient modification of pectin methylesterification remains poor. In cell wall mutants, the discrimination between the direct effects of the mutation on wall composition and those indirectly arising from signaling-related responses is always hard. However, a few clues indicate that some important signaling pathways could be activated by pectin methylesterification status during plant development. Auxin is known to regulate the DM of pectins (Voiniciuc et al., 2013; Schoenaers et al., 2018). Reciprocally, when HG de-methylesterification is inhibited, auxin signaling is altered in the shoot apical meristem by modification of the localization of the auxin transporter PIN1 (Braybrook and Peaucelle, 2013). More recently, pectin methylesterification was also shown to impact on auxin transport during apical hook formation, confirming the existence of a feedback loop between auxin and HG (Jonsson et al., 2021), the molecular basis of which remains unknown. Pectin methylesterification is also thought to be part of a feedback mechanism controlling polar changes in gene expression during leaf morphogenesis (Qi et al., 2017). Lastly, HG methylesterification regulates the production of a small cell-wall-derived dark signal involved in photomorphogenesis repression, which is required for skotomorphogenesis maintenance in etiolated seedlings (Sinclair et al., 2017).

In this context, we set out to determine the extent to which physiological modifications could originate from impairment in pectin methylesterification status, and to determine the impact this potential signaling function of pectin could have on plant development. For this purpose, we used the knockout pme36-1 mutant line, deficient for PME36 gene expression, in which pectin methylesterification status was strongly impaired in mature seeds. Interestingly, we discovered that several regulatory pathways were affected in the mutant, including hormone signaling, transcriptomic regulation and pectin remodeling, demonstrating that disruption in pectin methylesterification pattern can directly trigger large physiological changes. Surprisingly, however, these changes took place from seed maturation to seedling development, which is a kinetic exceeding the time required to only compensate the PME36 deficit in mature mutant seeds. More intriguingly, concomitantly to this long-lasting and dynamic physiological disorder, the mutant followed a developmental program, during germination and hypocotyl elongation, which was strictly the same as in the wild type. This led us to hypothesize 
that the physiological changes observed in pme36-1, far from being a disruption, actually revealed the existence of a homeostatic system connecting different regulatory pathways, which ensure the maintenance of the seed-to-seedling transition. This system was acting in the pme36-1 mutant by adjusting different physiological parameters, thereby restoring a new equilibrium suitable for maintaining normal developmental process in the mutant background.

\section{RESULTS \& DISCUSSION}

\section{PME36 is required to establish the demethylesterification pattern of $\mathrm{HG}$ in mature seeds}

Using RT-qPCR analysis in Col-0 plants and GUS staining of the promoter-reporter line, we assessed the expression pattern of the PECTIN METHYLESTERASE 36 (PME36) gene during seed maturation and early seedling development. The transcript level peaked in siliques from 13 to 16 days post-anthesis (DPA) (Figure 1A). A pool of PME36 mRNAs was stored in the mature seed, which rapidly decreased in 24 and 48 hours post-stratification (HPS) seedlings. In seeds and in 24 HPS seedlings, the promoter displayed constitutive activity, whereas in 48 HPS seedlings the GUS staining appeared stronger in cotyledons and in the upper part of the hypocotyl, indicating that residual gene expression was restricted to these organs at this latter stage (Figure 1B).

The high expression of PME36 during maturation suggested that the encoded protein might play an important role in the control of HG methylesterification in the seed. To address this question, we identified the pme36-1 mutant, a T-DNA insertion line that was a knock-out for PME36 gene expression. PME activity was significantly reduced in 16 DPA pme36-1 siliques compared to the wild type, indicating the importance of PME36 to the total PME activity during seed maturation (Figure 1C). In order to analyze whether the reduction of PME activity during seed maturation led to a concurrent increase in the level of methylesterified HG in mutant seed, we analyzed the methylesterification of pectins by enzymatic fingerprinting in mature seeds from pme36-1 and Col-0 (Voxeur et al., 2019; Hocq et al., 2020). The OG profile obtained, from HG hydrolysis by endo-polygalacturonase, revealed that more GalA3 and GalA4Me were released from pme36-1 than from Col-0 seeds, suggesting the presence of higher number of hydrolysable blocks of methylesterified HG in the mutant 
seed (Figure 1D). To characterize the pattern of HG methylesterification within the seed, sections of resin-embedded mature seeds from pme36-1 and Col-0 were probed with two different monoclonal antibodies directed to $\mathrm{HG}$ with specific patterns and degrees of methylesterification (DM). The antibody JIM7 was previously shown to display affinity for HG with medium DM and LM20 was characterized as preferentially binding to HG with medium to high DM (Clausen et al., 2003; Verhertbruggen et al., 2009). Labelling with Calcofluor White highlighted cell walls in medial longitudinal sections of Col-0 and pme36-1 mature seeds (Figures 1E and 1F). Higher magnification views of the pme36-1 sections allowed analysis of seed mucilage [M], endosperm [En] and embryo tissues [Em] (Figure 1G). JIM7 labelling revealed that HG with medium DM was restricted to the mucilage and the root vascular tissue in the wild-type mature seeds (Figure 1H). On the contrary, the JIM7 epitope was widely distributed in pme36-1 seeds, where it was abundant in embryo and endosperm as well as in the mucilage cells (Figures $\mathbf{1 I}$ and 1J). HG epitopes recognized by JIM7 are known to be partially sensitive to polygalacturonase treatment (Willats et al., 2000). The higher amount of GalA3 and GalA4Me released by the PG is therefore consistent with the increased JIM7 labelling observed in pme36-1 mature seed (Figure 1D). The LM20 epitope was only detected in the seed mucilage in Col-0, whereas it was present in the embryo, the mucilage and, to a lower extent, the endosperm of pme36-1 seeds, confirming the presence of HG of medium to high DM in the cell walls of mutant mature seeds (Figures $1 \mathrm{~K}$ to $1 \mathrm{M}$ ). Altogether, these results showed that, while Col-0 mature seeds contained a relative small amount of methylesterified $\mathrm{HG}$, this pattern was strongly impaired in mature seeds of pme36-1 with substantial enrichment in HG with medium to high DM. This indicates the crucial role PME36 plays in the orchestration of the demethylesterification of pectins in mature seeds of Arabidopsis.

Increased pectin methylesterification in pme36-1 seed is rapidly rectified and does not affect the germination process

In the course of seed development and during germination, cell wall loosening is required and could result from either a decrease or increase of the DM of HG. During Arabidopsis embryo morphogenesis, cell wall loosening is induced by the decrease of the DM of pectin, which is mediated by HMS protein (i.e. PME6) activity, allowing the necessary cell expansion for correct embryo shape and accumulation of storage reserves (Levesque-Tremblay et al., 2015a). In the hms-1 mutant, the increased DM 
of pectin generates cell wall stiffening leading to reduction of embryo cell size, which severely affects mutant seed development. Surprisingly, the increased DM of pectin did not lead to an equivalent alteration in seed morphology in pme36-1 compared to wild-type seed (Figures 1E and 1F). This is likely due to the different timing at which HMS and PME36 are expressed and, thus, by the difference in seed development stage when cells are subject to a higher DM in cell wall HG. In the wild type, HMS expression actually peaked in 7 DPA siliques, whereas PME36 expression reached its maximum 6 days later, in 13 DPA siliques. Therefore, while strongly disturbing seed development when arising during embryo morphogenesis, our results suggest that an increase in the DM of pectin does not produce the same effect when occurring during the subsequent maturation stage, as is the case for pme36-1.

During germination, the methylesterification of pectin was shown to play an opposite effect on cell wall plasticity than during embryo development. In Arabidopsis PMEI5 overexpressing seed, in which PME activity was strongly reduced, cell wall loosening was induced by the increase of pectin DM, which led to faster seed germination by facilitating testa rupture and radicle emergence through the endosperm (Müller et al., 2013). As a consequence, PME activity seems not to be necessary per se to achieve the germination process. However, specific regulation of PME activity was observed in Arabidopsis germinating seeds, peaking during testa rupture and then declining once endosperm rupture was reached. Moreover, analysis of testa rupture in garden cress seed added complexity into this model, highlighting specific regulations of $\mathrm{PME}$ expression occurring in different seed compartments, and also the antagonistic effects of PME treatment on testa rupture, which can be either positive or negative depending on the concentration of enzyme applied (Scheler et al., 2015). Altogether, these studies showed the crucial - although ambiguous - role of PME-related regulation of pectin DM in the germination process. In consequence, pme36-1 seeds were expected to germinate faster than the wild type, given its strong enrichment in methylesterified pectins, as in PMEI5 overexpressing seeds. We assessed the germination kinetics by determining the timing of testa and endosperm ruptures in post-stratification Col-0 and pme36-1 seeds. Surprisingly, the germination kinetic was the same in the mutant as in the wild type (Figure 1N). This unexpected result suggested that the strong defect in pectin methylesterification pattern observed in pme36-1 mature seed might have disappeared very quickly during the early germination stage, i.e. before testa rupture. To investigate this hypothesis, first we assessed the PME activity in germinating seeds 
from mutant and wild type. In 16 HPS seeds, before testa rupture stage, the PME activity in pme36-1 was already reestablished to the wild-type level, and in 40 HPS seed, when endosperm rupture stage was reached, the increase in PME activity level was the same in the mutant as in the wild type (Figure 10). This result indicated that the regulation of PME activity was fully restored in pme36-1 seed at very early stage of germination. The remaining question was to determine whether this recovery led to subsequent restoration of normal DM of pectin in the young seedling. To answer this, we analysed the methylesterification of pectin by enzymatic fingerprinting in 48 HPS mutant and wild-type seedlings. Despite the increase in GalA3 and GalA4Me previously observed in pme36-1 mature seed, in 48 HPS seedlings a similar OG profile was obtained for the two lines, indicating the mutant recovered a wild-type level of pectin DM during germination (Figure 1P). Overall, our results showed that, although pme36-1 mature seed displayed strong alterations in pectin methylesterification, the mutant rapidly regained a normal and steady ability to regulate pectin demethylesterification, before the testa rupture stage of germination. This led pme361 to recover a normal pectin methylesterification pattern and thus normal testa and endosperm ruptures, thus avoiding pectin-related effects on germination.

\section{Levels of PME and PMEI transcripts are strongly downregulated in pme36-1 mature seed}

The fast recovery in pme36-1 of PME activity to the wild-type level at a very early germination stage, i.e. in 16 HPS seed, led us to hypothesize the involvement of transcriptional regulation of $P M E$ and/or PMEI genes in the mutant during the seed-toseedling transition. Given the high sequence redundancy among both gene families and the possible variety of gene expression levels to be assessed, we designed a dedicated RT-qPCR assay allowing specific and sensitive quantification of transcripts from each of the 67 PME and 76 PMEl genes. By this means, quantification of individual PME and PMEI transcripts was performed in 13 DPA siliques, in mature seeds and in 24 HPS seeds from Col-0 and pme36-1 lines (Figure 2). The expression values shown for Col-0, represented with a red scale, are normalized non-calibrated RT-qPCR data, providing transcript levels that can be directly compared among genes. The relative expression values shown for pme36-1, are normalized RT-qPCR data that were calibrated to the wild-type level which was set to 1 (i.e. pme36-1/Col-0 expression ratio), providing fold-change levels of gene expression in the mutant compared to the 
wild type. This highlights the transcriptomic regulations triggered in the mutant, for which intensities are shown using a logarithmic bicolor scale, in red for upregulation and in blue for downregulation. Empty cells indicate absence of quantifiable transcripts. Transcript profiling of PMEl genes was very informative (Figure 2A). In Col-0 13 DPA siliques, 50 different PMEl genes were expressed, among which 46 remained expressed in the mature seed, where 20 maturation-specific transcripts were also produced. Therefore, mature seed turned out to contain a very rich pool of PMEI transcripts, from 66 out of the 76 PMEl genes identified in Arabidopsis. By contrast, the diversity of PMEI transcripts strongly decreased in 24 HPS seed, where only transcripts from 30 genes remained produced, among which there were no new specific genes. Translation from stored mRNAs has been shown for a long time as essential in the seed germination process and constitutes a major mechanism of protein production during early germination (Rajjou et al., 2004; Bai et al., 2020; Sano et al., 2020). Therefore, the importance of the pool of PMEI transcripts found in mature seeds, followed by the fast decay of this mRNA pool in 24 HPS seeds, strongly suggested the necessity for a high diversity of PMEI proteins to be transiently produced during seed imbibition. These PMEI presumably induce inhibition of PME activity during imbibition, which could be required to activate the germination process at the cell wall level. This hypothesis is supported by the positive impact PMEI5 overexpression played on germination activation (Müller et al., 2013).

In 13 DPA siliques, the expression levels of PMEl genes were not significantly different in pme36-1 compared to Col-0, indicating the absence of specific transcriptional regulation in the mutant at this stage (Figure 2A). On the contrary, the level of 54 out of the 66 expressed PMEI transcripts dramatically decreased in the pme36-1 mature seed, leading to a strong reduction in the PMEI transcript pool compared to the wild type. This PMEl transcriptional regulation was transitory, since expression of every gene returned to the wild-type level in 24 HPS pme36-1 seed, except for 4 of them, for which expression slightly increased. This slight overexpression, as for 2 other PMEI genes in the mutant mature seed, was likely to be due to homeostatic adjustment or a specific role for these PMEls, in response to the major alteration in PMEl gene expression occurring in pme36-1 mature seed. The fact remained that the main effect of PME36 disruption was a massive drop-down of PMEI transcript pool in the mutant mature seed, just as this pool raised its maximum in quantity and diversity in the wild type. Such a massive downregulation of gene expression is likely to trigger reduction 
of PMEI protein production at the onset of germination, thereby leading to increased PME activity by immediate release of PMEl-mediated PME inhibition. This would constitute an efficient compensatory mechanism that would explain by itself the fast reestablishment of PME activity and pectin methylesterification pattern to wild-type levels in pme36-1 germinating seed (Figures 10 and 1P).

Transcript profiling analysis provided a very different picture for PME genes (Figure 2B). While the expression level in Col-0 was constant for a few genes from 13 DPA siliques to 24 HPS seeds, most of PME genes displayed transcriptional regulation that was very specific to each developmental stage. This revealed a much more dynamic transcriptional regulation for PME than for PMEI genes during the seed-to-seedling transition. In 13 DPA siliques and 24 HPS seeds, no difference in PME transcript level was observed in pme36-1 compared to the wild type. On the contrary, the transcript level of 22 out of 45 expressed genes dropped in pme36-1 mature seeds. Thus, like for PMEls, but to a lower extent, the pool of PME transcripts decreased in pme36-1 mature seeds compared to the wild type. While the decrease of $P M E I$ transcript levels in pme36-1 mature seeds appeared to be consistent with the compensation of the PME activity defect, the concomitant decrease of PME transcript levels in the mutant was counterintuitive. Interestingly, a similar counteractive mechanism was recently described in the control of Arabidopsis gynecium development by ETTIN transcription factor (Andres-Robin et al., 2018). ETTIN induced PME activity by negatively regulating the expression of PMEI genes, thus decreasing the DM of pectin, which caused cell wall loosening required to promote gynecium development. However, at the same time, ETTIN also negatively regulated the expression of several PME genes (Andres-Robin et al., 2020). The authors hypothesized this simultaneous negative regulation of $P M E$ expression, the significance of which appeared unclear in their model, to be a part of a "gas and brake mechanism", in which antagonistic PMEI and $P M E$ genes were regulated in the same way in order to prevent excessive downstream effects. Our results confirm that such a mechanism is likely to exist and, by providing exhaustive expression data from both gene families, allow us to gain insight into the extent to which the transcriptional regulations of $P M E$ and PMEl genes might be connected.

Altogether, our results revealed the existence of a transcriptomic compensation in pme36-1 seeds, consisting on specific regulation of PME and PMEl gene expression during the maturation stage. This would trigger, at the time of germination, a fast 
recovery of PME activity and pectin methylesterification pattern to the wild-type level in the mutant. Since this compensation necessarily needs an upstream activation and should be fine-tuned to adapt pectin DM to the germination requirements, we postulated the existence of a more complex homeostatic system which involved other regulations ensuring a normal germination process in pme36-1 seeds. Such a system most likely requires crosstalk between different regulatory pathways, among which transcriptional regulation and hormone homeostasis are presumable players and for which further investigations were conducted in the pme36-1 mutant.

\section{pme36-1 displays dynamic gene expression regulation during germination}

Two transcriptional phases were previously characterized during Arabidopsis seed germination, separated by the testa rupture stage and displaying distinct transcriptome changes (Dekkers et al., 2013). In order to identify potential transcriptional regulations specifically occurring during pme36-1 germination, a transcriptomic analysis was performed in mutant and wild-type 16 and 24 HPS seeds, which characterized the stages of non-rupture seed imbibition and testa rupture, respectively (Figure 1N). We identified a set of 374 genes differentially expressed in the mutant compared to the wild type (Supplemental Dataset 1). Interestingly, while the developmental stages were spaced by only 8 hours, very few of the genes displayed the same transcription modulation (up- or down-regulation) in both conditions. The overwhelming majority showed either differential expression at only one stage or opposite regulation trends from one stage to another. This dynamic regulation exemplifies the specificity of the transcriptome at each transcriptional phase.

Three genes encoding polygacturonase (PG) proteins, among which PGAZAT that is involved in reproductive organ abscission in connection with abscisic acid (ABA) biosynthesis promotion (Ogawa et al., 2009; Xu et al., 2020), were deregulated in pme36-1 germinating seeds. PGs lead to cell wall loosening by catalyzing the degradation of pectic HG with low DM. In order to restore a normal level in germinating pme36-1 seeds, the DM of pectin necessarily decreased in the mutant from a very high level in the dry seed, to a wild-type level in 24 HPS seed. Interestingly, PG expression was upregulated in 16 HPS mutant seed, where pectins were probably still highly methylesterified, thus making them resistant to the lytic action of PGs. Then, in 24 HPS mutant seed, when pectins recovered a wild-type DM level and thereby an increased sensitivity to the degradation by PGs, $P G$ expression was downregulated. This 
transcriptional modulation suggested the possible existence of a regulatory process fine-tuning PG level according to the DM of pectin. Additional cell wall-related genes were differentially expressed in the mutant during germination, such as XTH4, XTH9 and $X$ TH25, which are involved in xyloglucan metabolism. Xyloglucans are cell wall components and their impairment can impact seed germination (Sechet et al., 2016; Shigeyama et al., 2016). Other genes differentially expressed in the mutant were related to seed germination regulation. For instance, AGAMOUS-LIKE67 expression was transiently strongly down-regulated in pme36-1 16 HPS seed. This gene was recently shown to inhibit seed germination under high temperature, by epigenetically activating the expression of SOMNUS gene encoding a zinc-finger protein ( $\mathrm{Li}$ et al., 2020). This example points out a possible connection between pectin methylesterification status and epigenetic regulations. This possibility remains to be evaluated and our goal here is neither to decipher the molecular mechanisms sustaining the transcriptional regulations found in pme36-1 nor to evaluate their impact, which would require further specific studies. We rather aim at identifying possible connections between pectin remodeling triggered by PME36 defect with other regulatory pathways that might be part of the compensatory system ensuring normal pme36-1 germination. For that purpose, we focused on the differentially expressed genes related to hormone signaling.

Several genes related to ABA signaling were deregulated in pme36-1. First, $P Y L 13$, which is a member of the $P Y L$ family of ABA receptors (Fuchs et al., 2014), and OST1, encoding an ABA-activated kinase belonging to the SNF1-related protein kinases (SnRK2) family (Vlad et al., 2009), were strongly downregulated in 16 HPS pme36-1 seeds. Both proteins being effective up-regulators of ABA signaling pathway, their down-regulation most likely led to decreased ABA signaling during pme36-1 seed imbibition. Moreover, the NCED9 gene, involved in ABA biosynthesis during seed development (Lefebvre et al., 2006), as well as PER1 that reduces seed germination by suppressing the ABA catabolism (Chen et al., 2020), were both downregulated in 16 HPS pme36-1 seeds, which might have an additive effect in decreasing ABA signaling through diminution of its levels in the mutant. A series of genes encoding typical ABA-induced seed proteins - like LEA, oleosin, oleosin-like, albumin and cruciferin proteins - were concurrently downregulated in 16 HPS mutant seeds, corroborating the possibility of ABA signaling reduction during mutant seed imbibition. However, at the same time, $H A / 3$ that encodes a PP2C ABA coreceptor inhibiting ABA 
signaling (Tischer et al., 2017) was down-regulated and RAP2-6, which overexpression has been shown to confer hypersensitivity to ABA signaling in seed germination (Zhu et al., 2010), was strongly up-regulated. Furthermore, the SASP gene, encoding a subtilase protein involved in reproductive development (Martinez et al., 2015) and in the regulation of ABA signaling (Wang et al., 2018), was also upregulated in 16 HPS mutant seeds. Finally, FAR1, which positively regulates the ABA pathway (Wang and Wang, 2015), was first up-regulated in 16 HPS pme36-1 seed and then down-regulated 8 hours later. Altogether, these transcriptional regulations pointed to transient and complex modulations of ABA signaling taking place during the early germination of pme36-1.

Interestingly, auxin signaling also appeared to be modulated in the mutant. First, JUMONJI13 (JMJ13), encoding a histone H3K27me3 demethylase, which was recently shown to regulate flowering time (Zheng et al., 2019) and self-fertility in Arabidopsis (Keyzor et al., 2021), was upregulated and then downregulated in pme361 seeds at 16 and 24 HPS, respectively. H3K27me3 is a repressive posttranslational histone modification which exerts epigenetic control of genes from the entire IAA signaling pathway (Lafos et al., 2011; He et al., 2012). By relieving the H3K27me3 repressive mark, JMJ13 is therefore likely to impact IAA signaling and its transcriptional modulation in pm36-1 might affect the hormone signaling during germination of the mutant. Second, CYP79B2 was upregulated in 16 HPS pme36-1 seeds. This gene encodes a key enzyme involved in the conversion of L-tryptophan (Trp) to IAOx (indole3-acetaldoxime), which is a common intermediate in Trp-dependent auxin biosynthesis pathway (reviewed in Casanova-Sáez et al. (2021)). Increase in IAOx levels result in elevated IAA biosynthesis and, thus, upregulation of CYP79B2 in 16 HPS pme36-1 seeds suggested that overproduction of the hormone arose in the mutant during seed imbibition. Nevertheless, IAOx is also a well-known precursor of indole glucosynolates (IGs), in a biosynthetic pathway for which the first step involves the SUR2 gene, encoding the cytochrome P450 CYP83B1 protein (Barlier et al., 2000; Bak and Feyereisen, 2001). This gene was precisely also upregulated in 16 HPS pme36-1 seed, thereby probably easing the potential IAA overproduction in the mutant seed to the benefit of the biosynthesis of IGs. Lastly, two genes encoding AUX/IAA transcriptional repressors, IAA2 and IAA33, were downregulated in 16 HPS pme36-1 seed. AUX/IAA proteins are auxin co-receptors and their degradation is crucial for auxin action (reviewed in Salehin et al. (2015)). Recently, IAA33 was shown to be a 
non-canonical AUX/IAA protein negatively regulating auxin signaling through competition with canonical AUX/IAA repressor IAA5 (Lv et al., 2020).

Overall, our results showed that pme36-1 seed displayed a highly dynamic transcriptional regulation at the outset of germination. As, several transcriptional regulations related to $A B A$ and auxin signaling were highlighted in the mutant, further investigations were conducted for the analysis of homeostasis and the role of these hormones during pme36-1 seed-to-seedling transition.

pme36-1 mature seeds contain reduced levels of ABA and IAA but display the same hormone sensitivity

ABA plays a major function in the regulation of dormancy and germination (reviewed in Shu et al. (2016)). Interestingly, during seed germination ABA treatment was shown to counteract the expected decline of PME activity by maintaining its high level beyond the testa rupture stage (Müller et al., 2013). In addition, the effect of the hormone was less efficient in delaying endosperm rupture in PMEI5 overexpressing seeds, where DM of pectin remained high due to PME activity inhibition. It was thus hypothesized that PME was involved in the ABA-dependent temporal regulation of endosperm rupture. However, the interplay between ABA and the DM of pectin in the control of seed germination remains poorly documented. To gain insight into this connection, we investigated the role of $\mathrm{ABA}$ during pme36-1 seed germination. First, $A B A$ quantification was performed in mature seeds as well as in 24 HPS and 48 HPS seedlings from Col-0 and pme36-1 lines. Interestingly, a strong reduction in ABA content was found in pme36-1 mature seeds compared to the wild type (Figure 3A). After germination, this difference decreased in the mutant, recovering to the wild-type ABA level in 48 HPS pme36-1 seedlings. The reduction in ABA content, at the outset of germination in the mutant, explained the decrease in ABA signaling suggested by the down-regulation of $P Y L 13$ expression as well as specific transcriptional regulations observed in 16 HPS mutant seeds, as previously detailed (Supplemental Dataset 1). To assess whether the sensitivity to ABA remained effective in the mutant, we analyzed the effect of hormone supplementation on pme36-1 and Col-0 germination in nonstratified seeds (Figure 3B). As expected, ABA treatment substantially delayed endosperm rupture in the wild type, but the same delay was observed in the mutant, indicating the hormone sensitivity was not affected in pme36-1 seeds. The decrease of the ABA level in pme36-1 mature seeds would be expected to constitute an effective 
signal, which should trigger early germination in mutant seeds. This effect should have been even more efficient due to its additive combination with the one expected from the high DM of pectin, inducing release of dormancy and premature germination in pme36-1 seed. But this was absolutely not the case, with pme36-1 following a wildtype germination kinetic, which added a new level of complexity in the crosstalk between $A B A$ homeostasis regulation and pectin methylesterification.

While ABA and GA are classically considered as the main hormones that antagonistically regulate seed dormancy and germination, over the past few years auxin has emerged as new master player acting in crosstalk with ABA (reviewed in Shu et al. (2016) and Matilla (2020)). To evaluate whether auxin homeostasis was impacted by the modification of pectin methylesterification in pme36-1, IAA quantification was performed in seeds and seedlings from pme36-1 and Col-0. Surprisingly, a strong decrease in IAA content was quantified in pme36-1 mature seeds (Figure 3C). After germination, the difference in IAA content between the mutant and the wild type decreased and remained negligible in 48 HPS seedlings. To determine whether the IAA decrease could affect the germination process in the mutant, we assessed pme36-1 and Col-0 germination under IAA supplementation (Figure 3D). IAA treatment did not affect the testa rupture and only slightly delayed the endosperm rupture in Col-0 and in pme36-1. The effect of IAA supplementation was less pronounced in the mutant, which makes sense considering the lower IAA content in the mutant, making probably the small effect produced by the supplementation less efficient in the mutant than in the wild type. The subtle negative effect of auxin on germination indicated that the IAA decrease observed in pme36-1 mature seeds might have either no effect on germination or only a small positive one. Either way, this effect was not comparable with the strong activation the decrease in ABA content was expected to exert on the germination of the mutant.

\section{Auxin homeostasis is modulated through increase of IAA conjugation in pme36- 1 seed}

The strong reduction of the IAA level in the mutant seeds and its subsequent fast recovery once germination had begun, were intriguing and suggested that a very specific regulation of auxin homeostasis occurred in the mutant. Conjugation of various amino acids to IAA, leading to inactivation or degradation, is a common way by which auxin homeostasis is modified, especially when dynamic and reversible modification 
of IAA concentration is required (Mellor et al., 2016; Casanova-Sáez et al., 2021). We quantified the level of two major IAA inactive conjugates, IAGlu (N-(Indole-3-ylAcetyl)Glutamate) and IAAsp (N-(Indole-3-ylAcetyl)-Aspartate), and the level of the oxidative form oxIAA that induces IAA degradation, in mature seeds as well as in 24 and 48 HPS seedlings from Col-0 and pme36-1 lines (Figure 3E to 3G). Interestingly, the levels of IAGlu, IAAsp and oxIAA were strongly increased in pme36-1 mature seeds and rapidly returned to the wild-type level during germination, except for IAGlu which remained at a higher level in the mutant seedlings compared to the wild type. These results indicated that the decrease of free IAA level observed in pme36-1 mature seeds and the fast recovery to the wild-type level during germination involved specific regulation of conjugate production and IAA degradation, highlighting the dynamic regulation of the auxin homeostasis occurring during the seed-to-seedling transition in the mutant. Auxin conjugation is catalyzed by acyl-acid-amido synthetases encoded by auxininduced GRETCHEN HAGEN 3 (GH3) genes belonging to a family of 20 genes in Arabidopsis (Staswick et al., 2005). We quantified the expression of the GH3 genes by RT-qPCR in pme36-1 mature seed (Figure 3H). While GH3.7 transcript content decreased, the quantity of GH3.3, GH3.4, GH3.5 and GH3.6 transcripts strongly increased in mature seeds of the mutant compared to the wild type, suggesting that increased IAA conjugation was sustained by transcriptional upregulation of these $\mathrm{GH} 3$ genes during pme36-1 seed maturation. The expression of $G H 3.3, G H 3.5$ and $G H 3.6$ genes were previously shown to be regulated by the AUXIN RESPONSE FACTOR genes $A R F 6$ and $A R F 8$, as positive regulators, and $A R F 17$, as negative regulator (Gutierrez et al., 2012). Interestingly, the expression of these 3 ARFs increased in 13 DPA pme36-1 siliques, ARF6 and ARF8 displaying a stronger upregulation than $A R F 17$, thereby providing a positive balance for subsequent activation of $\mathrm{GH} 3$ gene expression (Figure 3I). It is noteworthy that, PME36 expression increasing from 10 to 13 DPA siliques in the wild type, the defect in PME activity in the mutant cells was presumably perceived within this interval, which means that the gene expression modulation of the 3 ARFs in 13 DPA siliques was a very fast event. Therefore, the modulation of auxin homeostasis in pme36-1 seed, through transcriptomic regulation of the ARF/GH3 module, constituted an early response to PME36 disruption during seed maturation, occurring before the implementation of the compensatory PME and PMEl transcriptomic regulation. This most probably led to the decreased auxin content at the same time as the pectin methylesterification pattern was disrupted in the mutant 
seed. This concomitance suggested that the reduction of auxin content in pme36-1 seeds might be involved in setting up the transcriptomic PME/PMEI compensatory mechanism.

\section{The PME36 knockout is overcome by combined regulations ensuring normal germination kinetics}

Connections between the different regulatory pathways, deduced from modulations observed in pme36-1, are recapitulated in Figure $\mathbf{3} \mathbf{J}$ where consecutive steps are numbered. First, PME36 impairment negatively impacts on PME activity during seed maturation, which leads to an increase in the DM of pectins [1]. This triggers transcriptional activation of the ARF/GH3 module [2], leading to the reduction of IAA levels through increased conjugation activity [3]. Simultaneously, ABA content also decreases, which is possibly linked to the IAA level reduction given the connections previously shown between the signaling pathways of these two hormones during seed maturation and germination (Belin et al., 2009; Liu et al., 2013; Pellizzaro et al., 2020). Given the role of auxin in the regulation of cell wall-related genes (Majda and Robert, 2018), hormonal deregulation is likely to trigger subsequent transcriptional regulations in pme36-1 (i) on PME and PMEl genes during seed maturation [4] and (ii) on a wider set of genes during early germination [5], among which some genes related to hormone signaling. The drop in PMEI transcript level in mutant seeds is likely to reduce the quantity of neo-synthesized PMEI proteins during the imbibition stage, thus releasing PME inhibition at the outset of germination [6]. Concomitantly, the reduction in IAA levels, known to mediate cell wall acidification which triggers acid growth (Fendrych et al., 2016), probably leads to a transient increase of apoplast $\mathrm{pH}$ in imbibed mutant seeds [7]. This might constitute an additive positive effect, reducing PMEI-PME interactions for which stability declines when $\mathrm{pH}$ value rises to neutrality and, at the same time, initiating PME activity, which has an optimum at alkaline pH (Sénéchal et al., 2015; Hocq et al., 2017a, 2017b; Sénéchal et al., 2017). Moreover, a pH increase might also result in the reduction of $\mathrm{pH}$-dependent activity of wall-loosening agents like expansins (Cosgrove, 2016), thereby counterbalancing the potential loosening effect expected to promote germination acceleration - the high DM of pectins might cause in pme36-1 imbibed seeds and avoiding wall-loosening reactivation during the pectin demethylesterification process in germinating mutant seeds. Finally, enhanced PME activity allows a rapid decrease of the DM of pectin to wild-type levels in pme36-1 
young seedlings [8]. Overall, all these factors (modulation of the DM of pectins, transcriptome dynamics and hormone homeostasis) appeared to be accurately regulated to generate a physiological equilibrium that allows normal germination of the mutant seed. This sheds light on a previously undescribed regulatory network integrating different pathways, connected to pectin methylesterification status, to constitute a homeostatic system ensuring the seed-to-seedling transition.

\section{Unexpected shifted effects on jasmonate signaling and pectin remodeling take place in pme36-1 seedlings without affecting hypocotyl elongation}

In addition to auxin, GH3.3, GH3.5 and GH3.6 were also previously shown to conjugate jasmonate (JA) into inactive forms in hypocotyls from dark-grown Arabidopsis seedlings (Gutierrez et al., 2012). Considering the down-regulation of the three genes encoding these proteins in pme36-1 mature seeds (Figure $3 \mathrm{H}$ ), this raised questions as to whether JA homeostasis was also impaired in the mutant seeds. We quantified the level of both JA and jasmonoyl-L-isoleucine (JA-lle), the active signaling molecule recognized by the F-box receptor CORONATINE INSENSITIVE1 (COI1) (Fonseca et al., 2009), in mature seeds as well as in 24 and 48 HPS seedlings from Col-0 and pme36-1 lines (Figure 4A and 4B). In mature seeds, the JA-lle content was slightly lower in the mutant, whereas the JA level was the same in the mutant as in the wild type. However, in 24 and 48 HPS seedlings, JA and JA-lle levels strongly decreased in the mutant compared to the wild type. These JA homeostasis modifications occurred in pme36-1 when compensation of PME36 disruption was presumably overcome through the homeostatic system previously described in Figure 3J. Nevertheless, it meant that young pme36-1 seedlings were clearly still subject to physiological imbalance. While playing a major role in stress responses and in flower development (Browse and Wallis, 2019), JA is not known for controlling seed germination. But we checked whether JA signaling could impact pme36-1 germination, by assessing this process in mutant and wild-type seed under JA supplementation. The result showed no effect in both lines, confirming JA modulation was probably playing no role in the mutant germination (Supplemental Figure 1). In contrast, JA was recently shown to inhibit hypocotyl elongation in etiolated Arabidopsis seedlings (Zheng et al., 2017). In order to evaluate the impact JA/JA-lle deficiency could have on dark-grown hypocotyl elongation in pme36-1 seedlings, we conducted a phenotyping analysis of this developmental trait. Using a robotized camera and green LED illumination, we 
performed time-lapse imaging of vertical plates containing Col-0 and mutant seeds grown in the dark for 6 days. This experimental setup allowed individual tracking of each plant along the full developmental sequence, providing accurate measurement of hypocotyl elongation and, thus, of hypocotyl growth kinetics (Supplemental Video 1). Interestingly, during the 6 days of development in the dark, the hypocotyl growth kinetics were exactly the same in the mutant as in the wild type (Figure 4C). This result was surprising since an increase of hypocotyl elongation was expected in the mutant, at least at the beginning of the hypocotyl growth phase where decreased in JA/JA-Ile levels should have led to release the negative effect the hormone played on this developmental trait. To test whether the lack of developmental effect was due to a reduction in JA sensitivity in the mutant, we grew pme36-1 and wild-type seedlings in the dark under JA supplementation. In both lines, JA reduced hypocotyl elongation in the same way, confirming the effective sensitivity to JA in pme36-1 seedling and, thereby, keeping elusive the reason why reduced hormonal levels did not lead to more rapid elongation of the mutant hypocotyl (Supplemental Video 2). Another intriguing point is the shift observed between PME36 disruption, occurring during seed maturation and compensated afterward during early germination, with the decrease in JA content occurring in seedlings during the following developmental stage. Interestingly, a recent study demonstrated that turgor-driven alterations in cell walls stimulate JA-lle biosynthesis (Mielke et al., 2021). Accordingly, we hypothesized that the compensatory pectin remodeling, taking place in pme36-1 seeds during imbibition stage, which is precisely characterized by turgor pressure restoration, could transiently change cell wall mechanical properties in a way that could produce subsequent alterations in JA homeostasis. Conversely, the absence of a phenotype resulting from these alterations in pme36-1 dark-grown hypocotyl might suggest that the mutant cells displayed wall modifications that could counteract the hormonal effect. In order to detect such a cell wall remodeling in pme36-1 elongating hypocotyls, we performed a cell wall FTIR analysis on the hypocotyl in 72 HPS mutant and wild-type seedlings grown in the dark or under long-day condition (16h/8h). While no significant variation was shown in non-etiolated seedlings grown in the light, strong modifications in cell wall composition were shown in the mutant seedling grown in the dark (Figure 4D). Notably, the high positive peak at $1785 \mathrm{~cm}^{-1}$ for the Col-0/pme36-1 ratio clearly pointed out that pme36-1 hypocotyl displayed a drastic reduction in the level of methylesterified HG. Consistently, the high negative peak at $1630 \mathrm{~cm}^{-1}$ indicated a strong increase in 
de-methylesterified HG level in the mutant cell wall. Therefore, cell walls from pme361 etiolated hypocotyl turned out to contain pectins with lower DM than Col-0. This result appeared puzzling because de-methylesterification of pectins was shown to control the timing of the growth acceleration phase in dark-grown hypocotyls (Pelletier et al., 2010), whereas we showed here that the kinetics of hypocotyl elongation was not perturbed in pme36-1. In addition, the link between the original defect in PME36 expression during seed maturation and the shifted effect on cell wall remodeling at 72 HPS is enigmatic. However, a recent study showed that modification of pectin demethylesterification during seed maturation could have a long-term effect during further seedling development (Francoz et al., 2019), the early changes in pectin methylesterification status preparing cell walls for further changes. A similar shifted effect could be at work in the pme36-1 seedling. To conclude, the decrease in JA content as well as the de-methylesterification of $\mathrm{HG}$ should have triggered acceleration of hypocotyl elongation in pme36-1, but this was not the case. This indicates that other players are acting, which remain to be identified in order to complete our understanding of the homeostatic system reported in this study.

\section{Transcriptomic and hormonal regulations are triggered by pectin remodeling in a dynamic homeostatic system ensuring the seed-to-seedling transition}

Homeostatic systems are not easy to characterize since their main function is precisely to make silent transient variations that might occur during the life of an organism. When variations are too drastic, as for epistatic effects of a steady gene overexpression, homeostatic systems failed to maintain a physiological equilibrium, which most often leads to developmental defects. In this study, we took advantage of the strong, but transient, effect PME36 disruption had on pectin methylesterification in the mature seed. By analyzing the physiological perturbations induced in the mutant, in the absence of growth phenotypical effects, we unveiled the complexity of an undiscovered homeostatic system likely ensuring the maintenance of the seed-to-seedling transition by complex and long-lasting interactions between pectins, the transcriptome and hormones (Figure 4E). The picture we provide here is far from being complete. The signaling pathways, leading modulated pectin methylesterification levels to trigger transcriptome and hormonal changes, remain to be deciphered, along with the role played by other cell wall components. That being said, our work clearly shows that, beyond its mechanical role within the cell wall, pectin methylesterification status acts 
as an upstream modulator of diverse regulatory pathways involved in plant growth and development. With this in mind, effects of cell wall-related mutations should be analyzed as consequences of both the straightforward change in cell wall properties and also the modulation of signaling pathways impacting diverse regulatory networks.

\section{METHODS}

\section{Plant material and growth conditions}

The Arabidopsis pme36-1 mutant was isolated from the SALK T-DNA collection (SALK_022170, Col-0 ecotype). The left flanking sequences of the T-DNA insertion sites were amplified by PCR on genomic DNA using the TDNA specific primer LB (5'CGATTTCGGAACCACCATCAAACAGGA-3') and AtPME36 specific primers KOF (5'ACGGGACATAACGTTCGAGA-3') and KOR (5'-TCGATTTTCCTCCCTTTCG-3'). The mutant line was characterized by RT-PCR as knock-out for PME36 gene expression. Plants were grown on soil in a greenhouse under the following conditions: $16 \mathrm{~h}$ light $\left(120 \mu \mathrm{mol} \mathrm{m} \mathrm{m}^{-2} \mathrm{~s}^{-1}, 20^{\circ} \mathrm{C}\right) / 8 \mathrm{~h}$ dark $\left(15^{\circ} \mathrm{C}\right)$ cycles.

\section{Germination testing and hypocotyl elongation phenotyping}

Mature dry seeds were stored at room temperature for three months after harvesting and were then sterilized and sown in vitro as previously described (Gutierrez et al., 2009). Germination was analyzed under long-day conditions ( $16 \mathrm{~h} / 8 \mathrm{~h})$ at $20^{\circ} \mathrm{C}$. In vitro plates were transferred to the light to induce germination, which constitutes the time point $0 \mathrm{~h}$. Time measurement was defined as hour after induction (HAI), for seeds transferred just after sowing, and as hour post stratification (HPS), for seed transferred after a previous $48 \mathrm{~h}$-period in dark at $4^{\circ} \mathrm{C}$ for stratification. Germination was scored with a SteREO Discovery V20 stereomicroscope (Zeiss). Testa and endosperm ruptures were scored as in Müller et al. (2013). The effects of different hormones on germination were also assessed by adding $5 \mu \mathrm{M}$ of abscisic acid (ABA), $10 \mu \mathrm{M}$ of auxin (IAA) or $10 \mu \mathrm{M}$ of jasmonic acid (JA) (Sigma) in the medium. For each biological replicate, at least 50 seeds were analyzed and values in the graph are the mean from 3 biological replicates.

For analysis of hypocotyl growth kinetics, after germination induction in vitro plates were put in the dark at $21^{\circ} \mathrm{C}$ for 6 days in a Phenobox chamber. This specific growth chamber is designed to receive 27 square Petri dishes $\left(12 \mathrm{~cm}^{*} 12 \mathrm{~cm}\right)$ and allows automatic image acquisition of each plate using a 36 Mpix D810 camera (Nikon) fixed 
onto a robotic arm (Optimalog). Pictures were taken every $4 \mathrm{~h}$, during the first $20 \mathrm{~h}$, and then every $2 \mathrm{~h}$. Images from each seedling were analyzed by a specific software (Optimalog) for measurement of hypocotyl length. For each biological replicate, at least 90 hypocotyls were analyzed and values in the graph are the mean from 3 biological replicates.

\section{Analysis of promoter activity}

One kb of the promoter of PME36 was amplified from genomic DNA using AmpliTaq polymerase (Thermofisher) with the following primers (PrF 5'TCTAGATCCTTAGCAGGCGCAATTAT-3' And PrR CCCGGGAACAGCAAACGAAGAATGGAA-3'). The PCR product was cloned into the pGEM-T Easy vector (Promega), sequenced and then subcloned into the binary vector pBI101.3 (Clontech) upstream of the GUS coding sequence using Xbal/Smal restriction sites. Plant transformation, using Agrobacterium tumefaciens strain LBA4404, was performed according to the floral dip method (Clough and Bent, 1998). Transformants were selected on kanamycin at $80 \mu \mathrm{g} \mathrm{ml-1}$ and $\beta$-glucuronidase staining was carried out as previously described (Sessions et al., 1999), with $10 \mathrm{mM}$ $\mathrm{K} 3 \mathrm{Fe}(\mathrm{CN}) 6$ and $10 \mathrm{mM} \mathrm{K} 4 \mathrm{Fe}(\mathrm{CN}) 6$, to limit stain diffusion. Plant samples were destained in $75 \%(v / v)$ ethanol and images were acquired using a SteREO Discovery V20 stereomicroscope (Zeiss).

\section{RNA Isolation and cDNA Synthesis}

RNAs from pme36 and Col-0 siliques, seeds and seedlings were prepared as described previously (Gutierrez et al., 2006). cDNAs were synthesized by reverse transcribing $4 \mu \mathrm{g}$ of total RNA using Transcriptor High Fidelity cDNA synthesis kit (Roche) with oligo(dT)12 primer, according to the manufacturer's instructions. All cDNA samples were tested by PCR using specific primers flanking an intron sequence to confirm the absence of genomic DNA contamination.

\section{Real-time RT-PCR analysis}

Transcript levels were assessed by quantitative RT-PCR using a LightCycler 480 system (Roche), as described by Gutierrez et al. (2012). Real-Time RT-PCR data normalization was performed using TIP41 and APT1 as reference genes, which expression stability was firstly validated for the considered developmental stages, as required (Gutierrez et al., 2008). Sequence of primers for $G H 3$ and $A R F$ transcripts are 
available in Gutierrez et al. (2012). Primers sequences for PME and PMEI transcripts (Supplemental Table 1), designed towards the 3' end of the RNA sequence using QuantPrime (https://quantprime.mpimp-golm.mpg.de/) (Arvidsson et al., 2008) and Primer3 software, were validated by melting curve analysis and efficiency measurement.

\section{Affymetrix microarrays analysis}

16 and $24 \mathrm{~h}$ post-stratification seeds were collected, flash frozen and ground into liquid nitrogen. Total RNA extraction, RNA quantification and quality control, labelled singlestranded DNAs production, hybridization on Affymetrix four-arrays strips (Arabidopsis Gene 1.1 ST Array strip), imaging and data processing were performed as in Voxeur et al. (2019). Normalized expression values were filtered for statistical relevance of differential expression using FDR F-Test p-value $<0,01$ and are shown in Supplemental Dataset 1. Affymetrix Microarray data are available in the Gene expressionOmnibus database (https://www.ncbi.nlm.nih.gov/geo/query/acc.cgi?acc=GSE155433)

\section{Enzymatic fingerprinting of pectins}

Alcohol-insoluble material from ground seeds or hypocotyls was incubated during 16 $\mathrm{h}$ at $37^{\circ} \mathrm{C}$ in $50 \mathrm{mM}$ ammonium formate $\mathrm{pH} 5$ with 5 units of Aspergillus aculeatus endo-polygalacturonase (Megazyme), which hydrolyzed the HG between at least two non-esterified GalA residues and was tolerant to acetyl groups (Voxeur et al., 2019). The digestion products (oligogalacturonides, OGs) were separated according to their degrees of polymerisation and methylesterification using size exclusion chromatography (SEC) followed by mass spectrometry analysis, as previously described (Hocq et al., 2020).

\section{Fourier Transform-Infrared (FTIR) microspectroscopy analysis}

Dark and light grown three-day-old seedlings were squashed between two BaF2 windows and extensively rinsed in distilled water for $2 \mathrm{~min}$. The samples were then dried on the windows at $37^{\circ} \mathrm{C}$ for $20 \mathrm{~min}$. For each mutant, 20 spectra were collected either at the top or bottom part of individual hypocotyls of seedlings from 4 independent cultures (5 seedlings from each culture), as described by Mouille et al. (2003). Normalization of the data and method-test were performed as previously described (Mouille et al., 2003). 


\section{Extraction of protein fraction and determination of PME activity}

Samples were ground in liquid $\mathrm{N}_{2}$ to obtain a fine powder and then transferred in the extraction solution. Proteins were extracted in $20 \mathrm{mM}$ sodium phosphate buffer $\mathrm{pH} 7.5$, containing $1 \mathrm{M} \mathrm{NaCl}, 0.2 \%(\mathrm{w} / \mathrm{v})$ PVPP and $0.01 \%(\mathrm{v} / \mathrm{v})$ Tween 20 for $1 \mathrm{~h}$ at $4^{\circ} \mathrm{C}$ under shaking. Cellular fragments were pelleted by centrifugation at $12,000 \mathrm{~g}$ for $15 \mathrm{~min}$. Proteins from the supernatant were quantified by the Bradford method (Bradford, 1976) with a Protein Assay kit (Biorad). Protein extracts were assayed for total PME activity at $\mathrm{pH} 7.5$ using the alcohol oxidase coupled assay (Klavons and Bennett, 1986). Data are the means of 9 independent replicates. Data were statistically analyzed by a MannWhitney test (Statisca).

\section{Immunofluorescence microscopy}

Dry seeds were fixed in PEM buffer (50 mM PIPES, 5 mM EGTA, and 5 mM MgSO4, $\mathrm{pH}$ 6.9) containing $4 \%(\mathrm{w} / \mathrm{v})$ paraformaldehyde under vacuum $(1 \mathrm{~h}$ at room temperature). After two washes in in phosphate-buffered saline (PBS; $0.01 \mathrm{M}$ phosphate buffer, $0.0027 \mathrm{M}$ potassium chloride, and $0.137 \mathrm{M}$ sodium chloride, $\mathrm{pH} 7.4$ ), seeds were dehydrated and infiltrated with LR White resin (London Resin Company) as previously described (Lee et al., 2012). Sections were cut to a thickness of $0.5 \mu \mathrm{m}$ using a diamond knife on an Ultracut microtome (Reichart-Jung) and collected on multiwell slides (ICN Biomedicals) coated with Vectabond reagent (Vector Laboratories). Calcofluor staining and immunolocalisation with anti-HG antibodies JIM7 and LM20 was conducted as previously described (Lee et al., 2012). Epifluorescence and light microscopy analyses were performed on cross sections from at least three seeds.

\section{Quantification of phytohormones}

$50 \mathrm{mg}$ of fresh weight mature seeds, 24HPS and 48 HPS seedlings were harvested, dried on tissue paper and frozen in liquid nitrogen. The samples were prepared in three biological replicates and phytohormones were extracted using an aqueous solution of methanol $\left(10 \% \mathrm{MeOH} / \mathrm{H}_{2} \mathrm{O}, \mathrm{v} / \mathrm{v}\right)$ from $1 \mathrm{mg}$ of samples. The concentrations of endogenous auxin metabolites (free IAA, its conjugates IAAsp and IAGlu, and catabolite oxIAA), jasmonates (JA and JA-lle), and ABA were determined in three independent experiments, each composed of three biological replicates. A cocktail of stable isotope-labelled standards was added, 5 pmol of $\left[{ }^{13} \mathrm{C}_{6}\right]$ IAA, $\left[{ }^{13} \mathrm{C}_{6}\right]$ oxIAA, $\left[{ }^{2} \mathrm{H}_{5}\right]\left[{ }^{15} \mathrm{~N}\right] \mathrm{IAAsp}$, and $\left[{ }^{2} \mathrm{H} 5\right]\left[{ }^{15} \mathrm{~N}\right] \mathrm{IAGlu}, 10 \mathrm{pmol}$ of $\left[{ }^{2} \mathrm{H}_{6}\right] \mathrm{ABA},\left[{ }^{2} \mathrm{H}_{6}\right] J \mathrm{~A}$, and $\left[{ }^{2} \mathrm{H}_{2}\right] \mathrm{JA}-\mathrm{Ile}$, 
and $20 \mathrm{pmol}$ of $\left[{ }^{2} \mathrm{H}_{4}\right] \mathrm{SA}$ (all from Olchemim Ltd) per sample to validate the LC-MS/MS method using stable isotope dilution method. The extracts were purified using Oasis HLB columns (30 mg/1 ml, Waters) and targeted analytes were eluted using $80 \%$ $\mathrm{MeOH}$ according to the method previously described (Floková et al., 2014). Separation was performed on an Acquity UPLC ${ }^{\mathrm{TM}}$ System (Waters) equipped with an Acquity UPLC BEH C18 column (100 x $2.1 \mathrm{~mm}, 1.7 \mu \mathrm{m}$; Waters), and the effluent was introduced into the electrospray ion source of a triple quadrupole mass spectrometer Xevo ${ }^{\mathrm{TM}}$ TQ MS (Waters).

\section{ACKNOWLEDGMENTS}

This work was supported by a grant from the Agence Nationale de la Recherche (ANR09-BLANC-0007-01, GROWPEC project), by the Conseil Régional des Hauts-deFrance and by the Université de Picardie Jules Verne. J.P. acknowledges the funding from the Institut Universitaire de France (IUF). K.J.D.L. and J.P.K. acknowledge funding from the UK Biotechnology and Biosciences Research Council (BB/G024898/1) through the ERA-NET support of the vSeed programme. This work was also funded by the Ministry of Education, Youth and Sports of the Czech Republic (European Regional Development Fund-Project "Plants as a tool for sustainable global development" No. CZ.02.1.01/0.0/0.0/16_019/0000827).

\section{AUTHOR CONTRIBUTIONS}

F.J. and S.G. contributed equally to this work. F.J., S.B. and L.G. developed the RTqPCR assay on PME and PMEI genes, with the help of S.G., F.S., L.H. and Ga.M.; S.G., Ga.M, H.D. and L.G. performed the RT-qPCR analysis, the GUS assays, the PME activity quantification and the germination testing. H.D. performed the hypocotyl elongation phenotyping. S.G. and L.G. performed the microarrays analysis. P.A., M.S. and O.N. performed the hormone measurements. A.V., S.P. and S.V. developed and performed the enzymatic fingerprinting of pectins. K.J.D.L. and J.P.K. performed the immunofluorescence microscopy. Gr.M. performed the FTIR analysis. S.G., F.J., J.P. and L.G. designed the research and analyzed the data. L.G. and J.P. conceptualized and supervised the overall project. L.G. wrote the article with inputs from all co-authors. J.P.K. edited the article. 


\section{REFERENCES}

Altartouri, B., Bidhendi, A.J., Tani, T., Suzuki, J., Conrad, C., Chebli, Y., Liu, N., Karunakaran, C., Scarcelli, G., and Geitmann, A. (2019). Pectin chemistry and cellulose crystallinity govern pavement cell morphogenesis in a multi-step mechanism. Plant Physiol. 181: 127-141.

\section{Andres-Robin, A., Reymond, M.C., Brunoud, G., Martin-Magniette, M.L.,} Monéger, F., and Scutt, C.P. (2020). Immediate targets of ETTIN suggest a key role for pectin methylesterase inhibitors in the control of Arabidopsis gynecium development. Plant Signal. Behav. 15.

Andres-Robin, A., Reymond, M.C., Dupire, A., Battu, V., Dubrulle, N., Mouille, G., Lefebvre, V., Pelloux, J., Boudaoud, A., Traas, J., Scutt, C.P., and Monéger, F. (2018). Evidence for the regulation of gynoecium morphogenesis by ETTIN via cell wall dynamics. Plant Physiol. 178: 1222-1232.

Arvidsson, S., Kwasniewski, M., Riaño-Pachón, D.M., and Mueller-Roeber, B. (2008). QuantPrime - a flexible tool for reliable high-throughput primer design for quantitative PCR. BMC Bioinforma. 2008 91 9: 1-15.

Bai, B., Van Der Horst, S., Cordewener, J.H.G., America, T.A.H.P., Hanson, J., and Bentsink, L. (2020). Seed-stored mRNAs that are specifically associated to monosomes are translationally regulated during germination. Plant Physiol. 182: 378-392.

Bak, S. and Feyereisen, R. (2001). The involvement of two P450 enzymes, CYP83B1 and CYP83A1, in auxin homeostasis and glucosinolate biosynthesis. Plant Physiol. 127: 108-118.

Barlier, I., Kowalczyk, M., Marchant, A., Ljung, K., Bhalerao, R., Bennett, M., Sandberg, G., and Bellini, C. (2000). The SUR2 gene of Arabidopsis thaliana encodes the cytochrome P450 CYP83B1, a modulator of auxin homeostasis. Proc. Natl. Acad. Sci. U. S. A. 97: 14819-14824.

Belin, C., Megies, C., Hauserová, E., and Lopez-Molina, L. (2009). Abscisic acid represses growth of the arabidopsis embryonic axis after germination by enhancing auxin signalings. Plant Cell 21: 2253-2268. 
Bidhendi, A.J., Altartouri, B., Gosselin, F.P., and Geitmann, A. (2019).

Mechanical Stress Initiates and Sustains the Morphogenesis of Wavy Leaf Epidermal Cells. Cell Rep. 28: 1237-1250.e6.

Bradford, M. (1976). A Rapid and Sensitive Method for the Quantitation of Microgram Quantities of Protein Utilizing the Principle of Protein-Dye Binding. Anal. Biochem. 72: 248-254.

Braybrook, S.A. and Peaucelle, A. (2013). Mechano-Chemical Aspects of Organ Formation in Arabidopsis thaliana: The Relationship between Auxin and Pectin. PLoS One 8.

Browse, J. and Wallis, J.G. (2019). Arabidopsis flowers unlocked the mechanism of Jasmonate signaling. Plants 8.

Casanova-Sáez, R., Mateo-Bonmatí, E., and Ljung, K. (2021). Auxin metabolism in plants. Cold Spring Harb. Perspect. Med. 11: 1-23.

Chebli, Y., Bidhendi, A.J., Kapoor, K., and Geitmann, A. (2021). Cytoskeletal regulation of primary plant cell wall assembly. Curr. Biol. 31: R681-R695.

Chen, H., Ruan, J., Chu, P., Fu, W., Liang, Z., Li, Y., Tong, J., Xiao, L., Liu, J., Li, C., and Huang, S. (2020). AtPER1 enhances primary seed dormancy and reduces seed germination by suppressing the $A B A$ catabolism and $G A$ biosynthesis in Arabidopsis seeds. Plant J. 101: 310-323.

Clausen, M.H., Willats, W.G.T., and Knox, J.P. (2003). Synthetic methyl hexagalacturonate hapten inhibitors of anti-homogalacturonan monoclonal antibodies LM7, JIM5 and JIM7. Carbohydr. Res. 338: 1797-1800.

Clough, S.J. and Bent, A.F. (1998). Floral dip: A simplified method for Agrobacterium-mediated transformation of Arabidopsis thaliana. Plant J. 16: 735-743.

Cosgrove, D.J. (2016). Catalysts of plant cell wall loosening. F1000Research 5.

Cosgrove, D.J. (2018). Diffuse growth of plant cell walls. Plant Physiol. 176: 16-27.

Cosgrove, D.J. and Anderson, C.T. (2020). Plant Cell Growth: Do Pectins Drive Lobe Formation in Arabidopsis Pavement Cells? Curr. Biol. 30: R660-R662. 
Daher, F.B., Chen, Y., Bozorg, B., Clough, J., Jönsson, H., and Braybrook, S.A. (2018). Anisotropic growth is achieved through the additive mechanical effect of material anisotropy and elastic asymmetry. Elife 7.

Dekkers, B.J.W. et al. (2013). Transcriptional dynamics of two seed compartments with opposing roles in Arabidopsis seed germination. Plant Physiol. 163: 205215.

Du, J. et al. (2020). Mutations in the Pectin Methyltransferase QUASIMODO2 influence cellulose biosynthesis and wall integrity in arabidopsis. Plant Cell 32: 3576-3597.

Fendrych, M., Leung, J., and Friml, J. (2016). Tir1/AFB-Aux/IAA auxin perception mediates rapid cell wall acidification and growth of Arabidopsis hypocotyls. Elife 5.

Floková, K., Tarkowská, D., Miersch, O., Strnad, M., Wasternack, C., and Novák, O. (2014). UHPLC-MS/MS based target profiling of stress-induced phytohormones. Phytochemistry 105: 147-157.

Fonseca, S., Chico, J.M., and Solano, R. (2009). The jasmonate pathway: the ligand, the receptor and the core signalling module. Curr. Opin. Plant Biol. 12: 539-547.

Francis, K.E., Lam, S.Y., and Copenhaver, G.P. (2006). Separation of Arabidopsis pollen tetrads is regulated by QUARTET1, a pectin methylesterase gene. Plant Physiol. 142: 1004-1013.

Fuchs, S., Tischer, S. V., Wunschel, C., Christmann, A., and Grill, E. (2014). Abscisic acid sensor RCAR7/PYL13, specific regulator of protein phosphatase coreceptors. Proc. Natl. Acad. Sci. U. S. A. 111: 5741-5746.

Guénin, S. et al. (2011). Identification of pectin methylesterase 3 as a basic pectin methylesterase isoform involved in adventitious rooting in Arabidopsis thaliana. New Phytol. 192: 114-126.

Gutierrez, L., Bussell, J.D., Păcurar, D.I., Schwambach, J., Păcurar, M., and Bellini, C. (2009). Phenotypic plasticity of adventitious rooting in arabidopsis is controlled by complex regulation of AUXIN RESPONSE FACTOR transcripts 
and microRNA abundance. Plant Cell 21: 3119-3132.

Gutierrez, L., Conejero, G., Castelain, M., Guénin, S., Verdeil, J.L., Thomasset, B., and Van Wuytswinkel, O. (2006). Identification of new gene expression regulators specifically expressed during plant seed maturation. In Journal of Experimental Botany (J Exp Bot), pp. 1919-1932.

\section{Gutierrez, L., Mauriat, M., Pelloux, J., Bellini, C., and Van Wuytswinkel, 0.}

(2008). Towards a systematic validation of references in real-time RT-PCR. Plant Cell 20: 1734-1735.

Gutierrez, L., Mongelard, G., Floková, K., Păcurar, D.I., Novák, O., Staswick, P., Kowalczyk, M., Păcurar, M., Demailly, H., Geiss, G., and Bellini, C. (2012). Auxin controls Arabidopsis adventitious root initiation by regulating jasmonic acid homeostasis. Plant Cell 24: 2515-2527.

Haas, K.T., Wightman, R., Meyerowitz, E.M., and Peaucelle, A. (2020). Pectin homogalacturonan nanofilament expansion drives morphogenesis in plant epidermal cells. Science (80-. ). 367: 1003-1007.

He, C., Chen, X., Huang, H., and Xu, L. (2012). Reprogramming of H3K27me3 Is Critical for Acquisition of Pluripotency from Cultured Arabidopsis Tissues. PLoS Genet. 8.

Hocq, L. et al. (2017a). Combined experimental and computational approaches reveal distinct $\mathrm{pH}$ dependence of pectin Methylesterase Inhibitors. Plant Physiol. 173: 1075-1093.

Hocq, L. et al. (2020). The exogenous application of AtPGLR, an endopolygalacturonase, triggers pollen tube burst and repair. Plant J. 103: 617-633.

Hocq, L., Pelloux, J., and Lefebvre, V. (2017b). Connecting HomogalacturonanType Pectin Remodeling to Acid Growth. Trends Plant Sci. 22: 20-29.

Hongo, S., Sato, K., Yokoyama, R., and Nishitani, K. (2012).

Demethylesterification of the primary wall by PECTIN METHYLESTERASE35 provides mechanical support to the Arabidopsis stem. Plant Cell 24: 2624-2634.

Jonsson, K., Lathe, R.S., Kierzkowski, D., Routier-Kierzkowska, A.L., Hamant, O., and Bhalerao, R.P. (2021). Mechanochemical feedback mediates tissue 
bending required for seedling emergence. Curr. Biol. 31: 1154-1164.e3.

Keyzor, C., Mermaz, B., Trigazis, E., Jo, S.Y., and Song, J. (2021). Histone

Demethylases ELF6 and JMJ13 Antagonistically Regulate Self-Fertility in Arabidopsis. Front. Plant Sci. 12.

Klavons, J.A. and Bennett, R.D. (1986). Determination of Methanol Using Alcohol Oxidase and Its Application to Methyl Ester Content of Pectins. J. Agric. Food Chem. 34: 597-599.

Lafos, M., Kroll, P., Hohenstatt, M.L., Thorpe, F.L., Clarenz, O., and Schubert, D. (2011). Dynamic regulation of H3K27 trimethylation during arabidopsis differentiation. PLoS Genet. 7.

Lee, K.J.D., Dekkers, B.J.W., Steinbrecher, T., Walsh, C.T., Bacic, A., Bentsink, L., Leubner-Metzger, G., and Paul Knox, J. (2012). Distinct cell wall architectures in seed endosperms in representatives of the Brassicaceae and Solanaceae. Plant Physiol. 160: 1551-1566.

Lefebvre, V., North, H., Frey, A., Sotta, B., Seo, M., Okamoto, M., Nambara, E., and Marion-Poll, A. (2006). Functional analysis of Arabidopsis NCED6 and NCED9 genes indicates that ABA synthesized in the endosperm is involved in the induction of seed dormancy. Plant J. 45: 309-319.

Leroux, C. et al. (2015). PECTIN METHYLESTERASE48 is involved in arabidopsis pollen grain germination. Plant Physiol. 167: 367-380.

Levesque-Tremblay, G., Müller, K., Mansfield, S.D., and Haughn, G.W. (2015a). Highly methyl esterified seeds is a pectin methyl esterase involved in embryo development. Plant Physiol. 167: 725-737.

Levesque-Tremblay, G., Pelloux, J., Braybrook, S.A., and Müller, K. (2015b). Tuning of pectin methylesterification: consequences for cell wall biomechanics and development. Planta 242: 791-811.

Li, P., Zhang, Q., He, D., Zhou, Y., Ni, H., Tian, D., Chang, G., Jing, Y., Lin, R., Huang, J., and Hu, X. (2020). AGAMOUS-LIKE67 Cooperates with the Histone Mark Reader EBS to Modulate Seed Germination under High Temperature. Plant Physiol. 184: 529-545. 
Liu, X., Zhang, H., Zhao, Y., Feng, Z., Li, Q., Yang, H.Q., Luan, S., Li, J., and He, Z.H. (2013). Auxin controls seed dormancy through stimulation of abscisic acid signaling by inducing ARF-mediated ABI3 activation in Arabidopsis. Proc. Natl. Acad. Sci. U. S. A. 110: 15485-15490.

Lv, B. et al. (2020). Non-canonical AUX / IAA protein IAA 33 competes with canonical AUX / IAA repressor IAA 5 to negatively regulate auxin signaling . EMBO J. 39.

Majda, M., Grones, P., Sintorn, I.M., Vain, T., Milani, P., Krupinski, P., ZagórskaMarek, B., Viotti, C., Jönsson, H., Mellerowicz, E.J., Hamant, O., and Robert, S. (2017). Mechanochemical Polarization of Contiguous Cell Walls Shapes Plant Pavement Cells. Dev. Cell 43: 290-304.e4.

Majda, M. and Robert, S. (2018). The role of auxin in cell wall expansion. Int. J. Mol. Sci. 19.

Martinez, D.E., Borniego, M.L., Battchikova, N., Aro, E.M., Tyystjärvi, E., and Guiamét, J.J. (2015). SASP, a Senescence-Associated Subtilisin Protease, is involved in reproductive development and determination of silique number in Arabidopsis. J. Exp. Bot. 66: 161-174.

Matilla, A.J. (2020). Auxin: Hormonal signal required for seed development and dormancy. Plants 9: 1-17.

Mellor, N. et al. (2016). Dynamic regulation of auxin oxidase and conjugating enzymes AtDAO1 and GH3 modulates auxin homeostasis. Proc. Natl. Acad. Sci. U. S. A. 113: 11022-11027.

Mielke, S., Zimmer, M., Meena, M.K., Dreos, R., Stellmach, H., Hause, B., Voiniciuc, C., and Gasperini, D. (2021). Jasmonate biosynthesis arising from altered cell walls is prompted by turgor-driven mechanical compression. Sci. Adv. 7.

Mouille, G., Robin, S., Lecomte, M., Pagant, S., and Höfte, H. (2003). Classification and identification of Arabidopsis cell wall mutants using FourierTransform InfraRed (FT-IR) microspectroscopy. Plant J. 35: 393-404. 
Usadel, B., Haughn, G., and Kermode, A.R. (2013). Demethylesterification of cell wall pectins in Arabidopsis plays a role in seed germination. Plant Physiol. 161: 305-316.

Ogawa, M., Kay, P., Wilson, S., and Swain, S.M. (2009). Arabidopsis Dehiscence Zone Polygalacturonase1 (ADPG1), ADPG2, and Quartet2 are polygalacturonases required for cell separation during reproductive development in Arabidopsis. Plant Cell 21: 216-233.

Peaucelle, A., Braybrook, S.A., Le Guillou, L., Bron, E., Kuhlemeier, C., and Höfte, H. (2011a). Pectin-induced changes in cell wall mechanics underlie organ initiation in Arabidopsis. Curr. Biol. 21: 1720-1726.

Peaucelle, A., Louvet, R., Johansen, J.N., Höfte, H., Laufs, P., Pelloux, J., and Mouille, G. (2008). Arabidopsis Phyllotaxis Is Controlled by the MethylEsterification Status of Cell-Wall Pectins. Curr. Biol. 18: 1943-1948.

Peaucelle, A., Louvet, R., Johansen, J.N., Salsac, F., Morin, H., Fournet, F., Belcram, K., Gillet, F., Höfte, H., Laufs, P., Mouille, G., and Pelloux, J. (2011b). The transcription factor BELLRINGER modulates phyllotaxis by regulating the expression of a pectin methylesterase in Arabidopsis. Development 138: 4733-4741.

Peaucelle, A., Wightman, R., and Höfte, H. (2015). The Control of Growth Symmetry Breaking in the Arabidopsis Hypocotyl. Curr. Biol. 25: 1746-1752.

Pelletier, S. et al. (2010). A role for pectin de-methylesterification in a developmentally regulated growth acceleration in dark-grown Arabidopsis hypocotyls. New Phytol. 188: 726-739.

Pellizzaro, A., Neveu, M., Lalanne, D., Ly Vu, B., Kanno, Y., Seo, M., Leprince, O., and Buitink, J. (2020). A role for auxin signaling in the acquisition of longevity during seed maturation. New Phytol. 225: 284-296.

Qi, J., Wu, B., Feng, S., Lü, S., Guan, C., Zhang, X., Qiu, D., Hu, Y., Zhou, Y., Li, C., Long, M., and Jiao, Y. (2017). Mechanical regulation of organ asymmetry in leaves. Nat. Plants 3: 724-733.

Rajjou, L., Gallardo, K., Debeaujon, I., Vandekerckhove, J., Job, C., and Job, D. 
(2004). The effect of $\alpha$-amanitin on the arabidopsis seed proteome highlights the distinct roles of stored and neosynthesized mRNAs during germination. Plant Physiol. 134: 1598-1613.

Salehin, M., Bagchi, R., and Estelle, M. (2015). ScfTIR1/AFB-based auxin perception: Mechanism and role in plant growth and development. Plant Cell 27: 9-19.

Sampathkumar, A. (2020). Mechanical feedback-loop regulation of morphogenesis in plants. Dev. 147.

Sano, N., Rajjou, L., and North, H.M. (2020). Lost in Translation: Physiological Roles of Stored mRNAs in Seed Germination. Plants (Basel, Switzerland) 9.

Scheler, C. et al. (2015). Promotion of testa rupture during garden cress germination involves seed compartment-specific expression and activity of pectin methylesterases. Plant Physiol. 167: 200-215.

Schoenaers, S. et al. (2018). The Auxin-Regulated CrRLK1L Kinase ERULUS Controls Cell Wall Composition during Root Hair Tip Growth. Curr. Biol. 28: 722732.e6.

Sechet, J., Frey, A., Effroy-Cuzzi, D., Berger, A., Perreau, F., Cueff, G., Charif, D., Rajjou, L., Mouille, G., North, H.M., and Marion-Poll, A. (2016). Xyloglucan metabolism differentially impacts the cell wall characteristics of the endosperm and embryo during Arabidopsis seed germination. Plant Physiol. 170.

Sénéchal, F. et al. (2015). Tuning of pectin methylesterification: Pectin methylesterase inhibitor 7 modulates the processive activity of co-expressed pectin methylesterase 3 in a pH-dependentmanner. J. Biol. Chem. 290: 2332023335.

Sénéchal, F., Habrylo, O., Hocq, L., Domon, J.M., Marcelo, P., Lefebvre, V., Pelloux, J., and Mercadante, D. (2017). Structural and dynamical characterization of the $\mathrm{pH}$-dependence of the pectin methylesterase-pectin methylesterase inhibitor complex. J. Biol. Chem. 292: 21538-21547.

Sessions, A., Weigel, D., and Yanofsky, M.F. (1999). The Arabidopsis thaliana MERISTEM LAYER 1 promoter specifies epidermal expression in meristems and 
young primordia. Plant J. 20: 259-263.

Shigeyama, T., Watanabe, A., Tokuchi, K., Toh, S., Sakurai, N., Shibuya, N., and Kawakami, N. (2016). a-Xylosidase plays essential roles in xyloglucan remodelling, maintenance of cell wall integrity, and seed germination in Arabidopsis thaliana. J. Exp. Bot. 67: 5615-5629.

Shu, K., Liu, X.D., Xie, Q., and He, Z.H. (2016). Two Faces of One Seed: Hormonal Regulation of Dormancy and Germination. Mol. Plant 9: 34-45.

Sinclair, S.A., Larue, C., Bonk, L., Khan, A., Castillo-Michel, H., Stein, R.J., Grolimund, D., Begerow, D., Neumann, U., Haydon, M.J., and Krämer, U. (2017). Etiolated Seedling Development Requires Repression of Photomorphogenesis by a Small Cell-Wall-Derived Dark Signal. Curr. Biol. 27: 3403-3418.e7.

Staswick, P.E., Serban, B., Rowe, M., Tiryaki, I., Maldonado, M.T., Maldonado, M.C., and Suza, W. (2005). Characterization of an arabidopsis enzyme family that conjugates amino acids to indole-3-acetic acid. Plant Cell 17: 616-627.

Tian, G.W., Chen, M.H., Zaltsman, A., and Citovsky, V. (2006). Pollen-specific pectin methylesterase involved in pollen tube growth. Dev. Biol. 294: 83-91.

Tischer, S. V., Wunschel, C., Papacek, M., Kleigrewe, K., Hofmann, T., Christmann, A., and Grill, E. (2017). Combinatorial interaction network of abscisic acid receptors and coreceptors from Arabidopsis thaliana. Proc. Natl. Acad. Sci. U. S. A. 114: 10280-10285.

Turbant, A., Fournet, F., Lequart, M., Zabijak, L., Pageau, K., Bouton, S., and Van Wuytswinkel, O. (2016). PME58 plays a role in pectin distribution during seed coat mucilage extrusion through homogalacturonan modification. J. Exp. Bot. 67: 2177-2190.

Vaahtera, L., Schulz, J., and Hamann, T. (2019). Cell wall integrity maintenance during plant development and interaction with the environment. Nat. Plants 5 : 924-932.

Verhertbruggen, Y., Marcus, S.E., Haeger, A., Ordaz-Ortiz, J.J., and Knox, J.P. (2009). An extended set of monoclonal antibodies to pectic homogalacturonan. 
Carbohydr. Res. 344: 1858-1862.

Vlad, F., Rubio, S., Rodrigues, A., Sirichandra, C., Belin, C., Robert, N., Leung, J., Rodriguez, P.L., Laurière, C., and Merlot, S. (2009). Protein phosphatases $2 \mathrm{C}$ regulate the activation of the Snf1-related kinase OST1 by abscisic acid in Arabidopsis. Plant Cell 21: 3170-3184.

Voiniciuc, C., Dean, G.H., Griffiths, J.S., Kirchsteiger, K., Hwang, Y.T., Gillett, A., Dow, G., Western, T.L., Estelle, M., and Haughn, G.W. (2013). Flying saucer1 is a transmembrane RING E3 ubiquitin ligase that regulates the degree of pectin methylesterification in Arabidopsis seed mucilage. Plant Cell 25: 944-959.

Voxeur, A. et al. (2019). Oligogalacturonide production upon Arabidopsis thalianaBotrytis cinerea interaction. Proc. Natl. Acad. Sci. U. S. A. 116: 19743-19752.

Wang, H. and Wang, H. (2015). Multifaceted roles of FHY3 and FAR1 in light signaling and beyond. Trends Plant Sci. 20: 453-461.

Wang, M., Yuan, D., Gao, W., Li, Y., Tan, J., and Zhang, X. (2013). A Comparative Genome Analysis of PME and PMEI Families Reveals the Evolution of Pectin Metabolism in Plant Cell Walls. PLoS One 8.

Wang, Q. et al. (2018). Arabidopsis subtilase SASP is involved in the regulation of ABA signaling and drought tolerance by interacting with OPEN STOMATA 1. J. Exp. Bot. 69: 4403-4417.

Willats, W.G.T., Limberg, G., Buchholt, H.C., Van Alebeek, G.J., Benen, J., Christensen, T.M.I.E., Visser, J., Voragen, A., Mikkelsen, J.D., and Knox, J.P. (2000). Analysis of pectic epitopes recognised by hybridoma and phage display monoclonal antibodies using defined oligosaccharides, polysaccharides, and enzymatic degradation. Carbohydr. Res. 327: 309-320.

Willats, W.G.T., Orfila, C., Limberg, G., Buchholt, H.C., Van Alebeek, G.J.W.M., Voragen, A.G.J., Marcus, S.E., Christensen, T.M.I.E., Mikkelsen, J.D., Murray, B.S., and Knox, J.P. (2001). Modulation of the degree and pattern of methyl-esterification of pectic homogalacturonan in plant cell walls: Implications for pectin methyl esterase action, matrix properties, and cell adhesion. J. Biol. Chem. 276: 19404-19413. 
Wolf, S. (2017). Plant cell wall signalling and receptor-like kinases. Biochem. J. 474: 471-492.

Xu, P., Chen, H., and Cai, W. (2020). Transcription factor CDF4 promotes leaf senescence and floral organ abscission by regulating abscisic acid and reactive oxygen species pathways in Arabidopsis . EMBO Rep. 21.

Zheng, S. et al. (2019). The Arabidopsis H3K27me3 demethylase JUMONJI 13 is a temperature and photoperiod dependent flowering repressor. Nat. Commun. 10.

Zheng, Y., Cui, X., Su, L., Fang, S., Chu, J., Gong, Q., Yang, J., and Zhu, Z. (2017). Jasmonate inhibits COP1 activity to suppress hypocotyl elongation and promote cotyledon opening in etiolated Arabidopsis seedlings. Plant J. 90: 1144-1155.

Zhu, Q., Zhang, J., Gao, X., Tong, J., Xiao, L., Li, W., and Zhang, H. (2010). The Arabidopsis AP2/ERF transcription factor RAP2.6 participates in ABA, salt and osmotic stress responses. Gene 457: 1-12. 


\section{FIGURES}

A
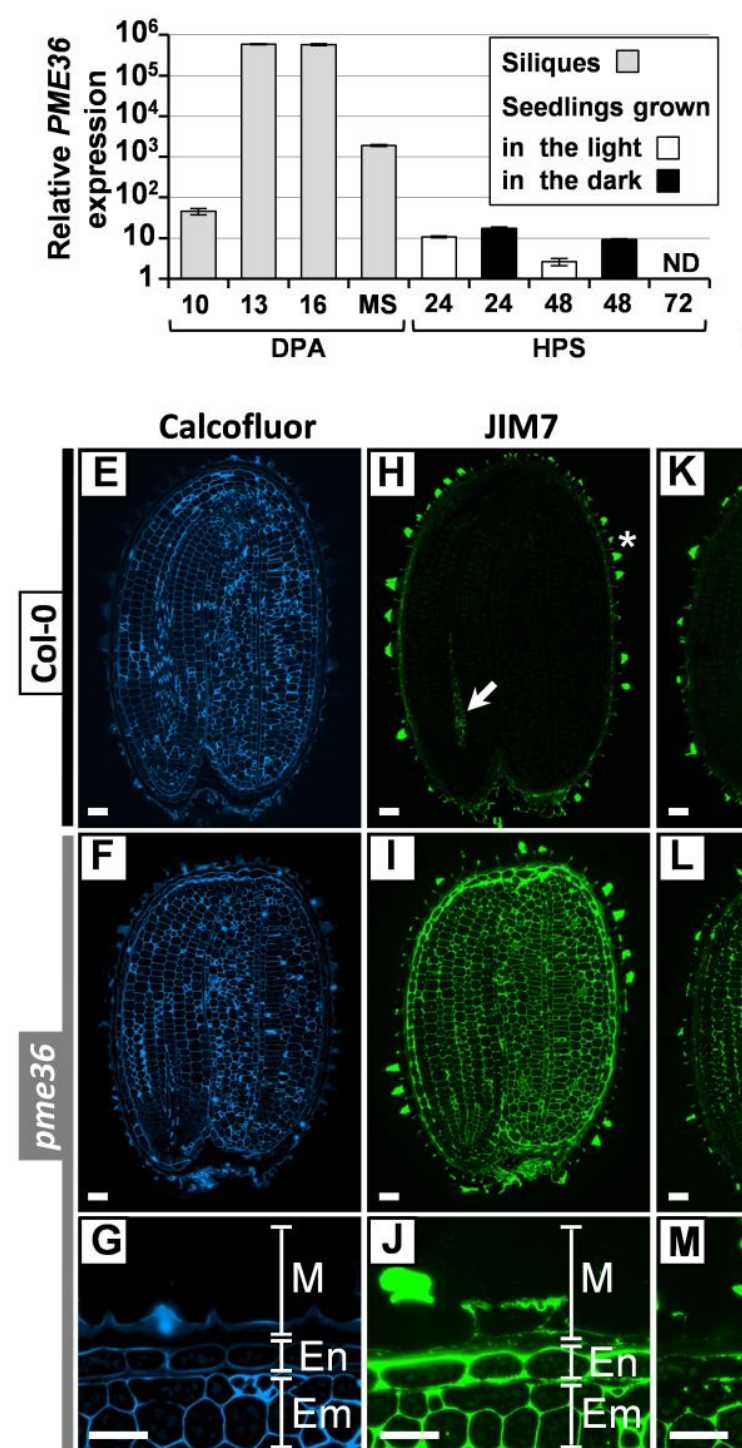

B

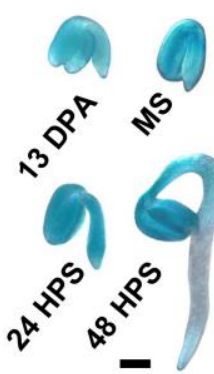

LM20
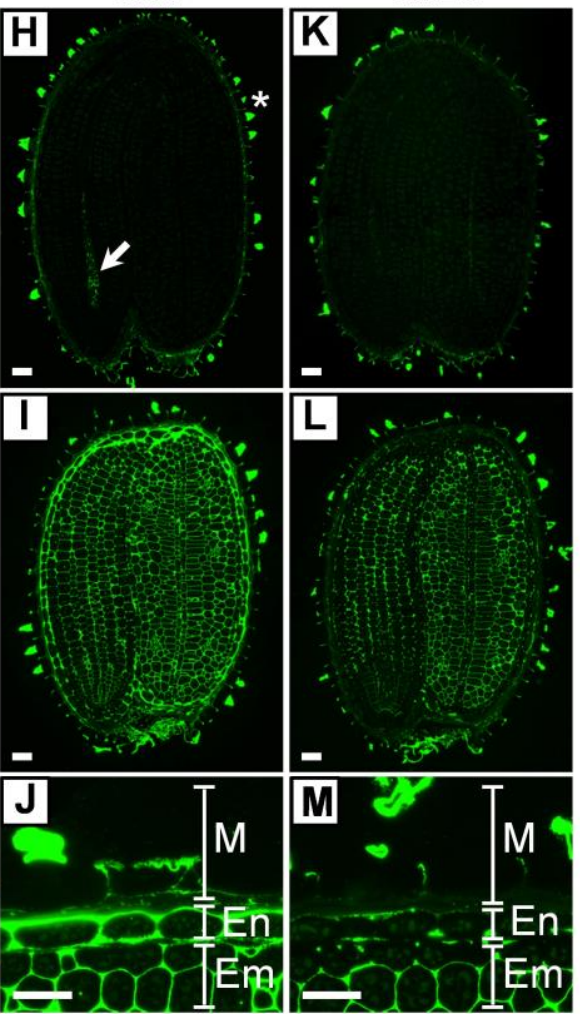

C

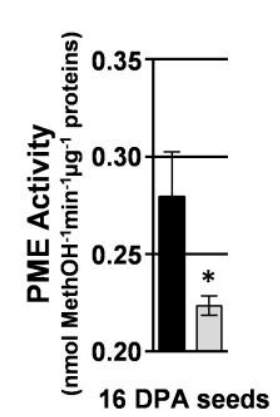

Colo pme36

D

$\mathbf{N}$
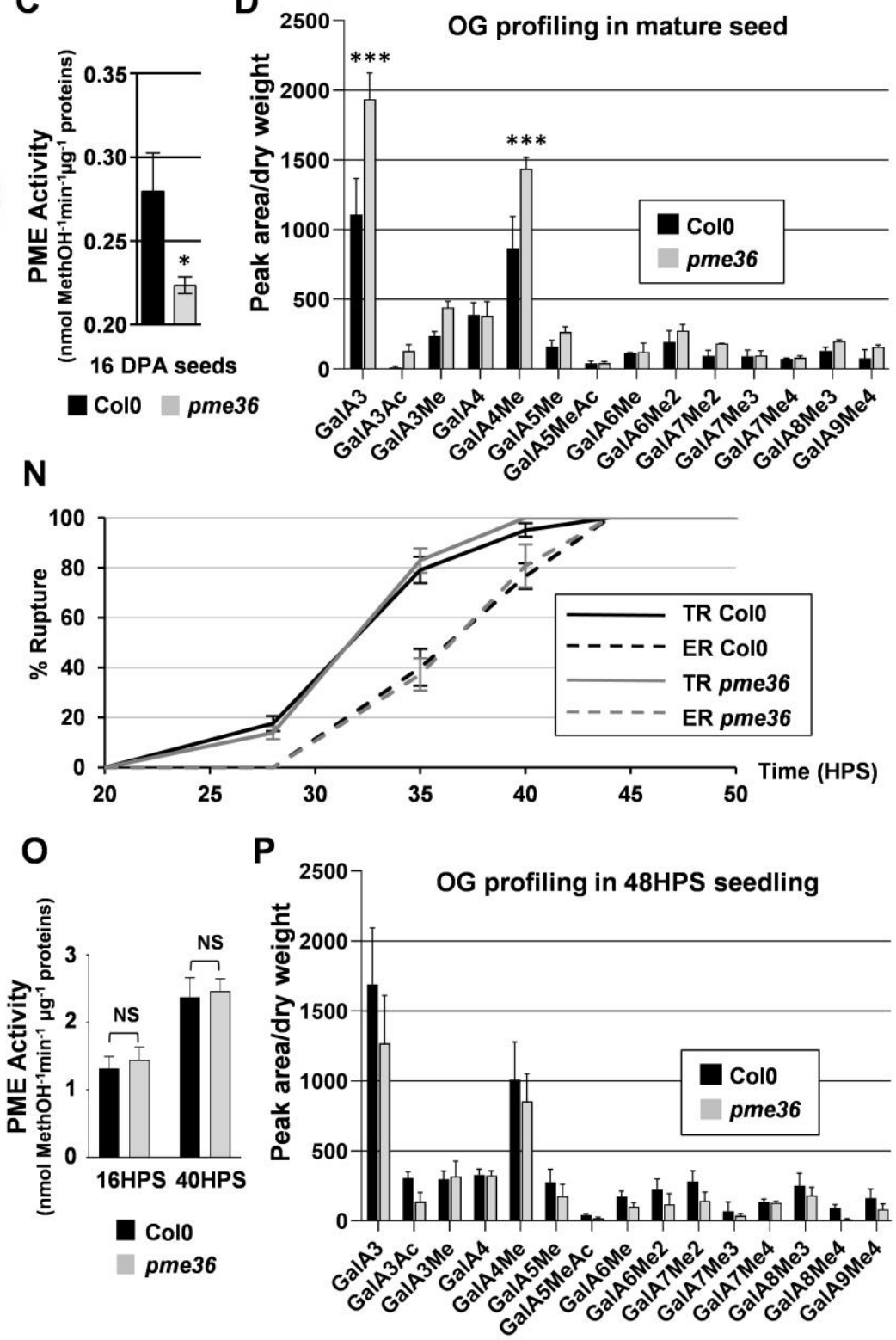

Figure 1. Impaired pectin demethylesterification in pme36-1 seed is rapidly rectified and does not affect the germination process.

(A) Quantification by RT-qPCR of PME36 transcripts in 10, 13 and 16 days post anthesis (DPA) Col-0 siliques, in mature seeds (MS) and in 24 and 48 hours post stratification (HPS) Col-0 seedlings grown in long-day conditions or in the dark. In 72 HPS Col-0 seedlings, no PME36 transcripts were detected whatever the growth conditions. Error bars indicate +/- SE obtained from 3 qPCR replicates (B) GUS staining of promPME36:GUS in 13 DPA seeds, in mature seeds (MS) and in 24 and 48 HPS seedlings grown in the dark. Bar $=200 \mu \mathrm{m}$ (C) PME activity was assessed in Col-0 and pme36-1 16 DPA seeds. A Mann-Whitney Comparison Test indicated that PME activity was significantly reduced in pme36-1 16 DAF seeds $\left({ }^{*}\right)$ compared to the wild-type $(\mathrm{P}<0.05)$. Error bars indicate $\mathrm{SE}$ obtained from three independent biological replicates, each one composed of three technical replicates. (D) Enzymatic fingerprinting of pectins from mature Col-0 and pme36-1 seed. Sidak's multiple comparisons test determined that significantly higher quantities of GalA3 and 
GalA4Met were released from pme36-1 seed than from Col-0 $\left(^{* \star *}\right)$. (E) to (M) Medial sections of resin-embedded Col-0 and pme36-1 mature seeds stained by Calcofluor White (E-G), which reveals all cell walls, and labelled with monoclonal antibodies JIM7 (H-J) and LM20 (K-M), which target HG with specific patterns and degrees of methylesterification. $(\mathrm{G}),(\mathrm{J}),(\mathrm{M})$ show magnification of a part of the seed tegument from (F), (I), (L) sections, respectively. (H) In Col-0 seeds, partially methylesterified HG recognised by JIM7 was restricted to mucilage $\left({ }^{*}\right)$ and root vascular tissue (arrow). (I) and (J) The JIM7 epitope was abundant in embryo, endosperm and mucilage of pme36-1 seeds. (K) The LM20 epitope was restricted to the seed testa/mucilage in Col-0. (L) and (M) The LM20 epitope was present in the embryo, mucilage and, at lower levels, endosperm of pme36-1 seeds. Em: embryo; En: endosperm; M: mucilage. Scale bars $=25 \mu \mathrm{m}$. (N) Seed germination kinetic of pme36-1 and Col-0 seeds characterized by testa rupture (TR) and endosperm rupture (ER). Data represent averages and error bars indicate $+/-$ SE, obtained from three independent biological replicates of at least 50 seeds each. (O) PME activity was assessed in Col0 and pme36-1 16 and 40 HPS seeds. A Mann-Whitney Comparison Test indicated that PME activity was not significantly different in pme36-1 compared to the wild-type $(P<0.05)$. Error bars indicate SE obtained from three independent biological replicates, each one composed of three technical replicates. (P) Enzymatic fingerprinting of pectins from 48 HPS Col-0 and pme36-1 seedling, showing no difference between the two lines. 
bioRxiv preprint doi: $\mathrm{https}$ ://doi org/10.1101/2021.07 22.453319: this version posted July 23, 2021. The copyright holder for this preprint

A

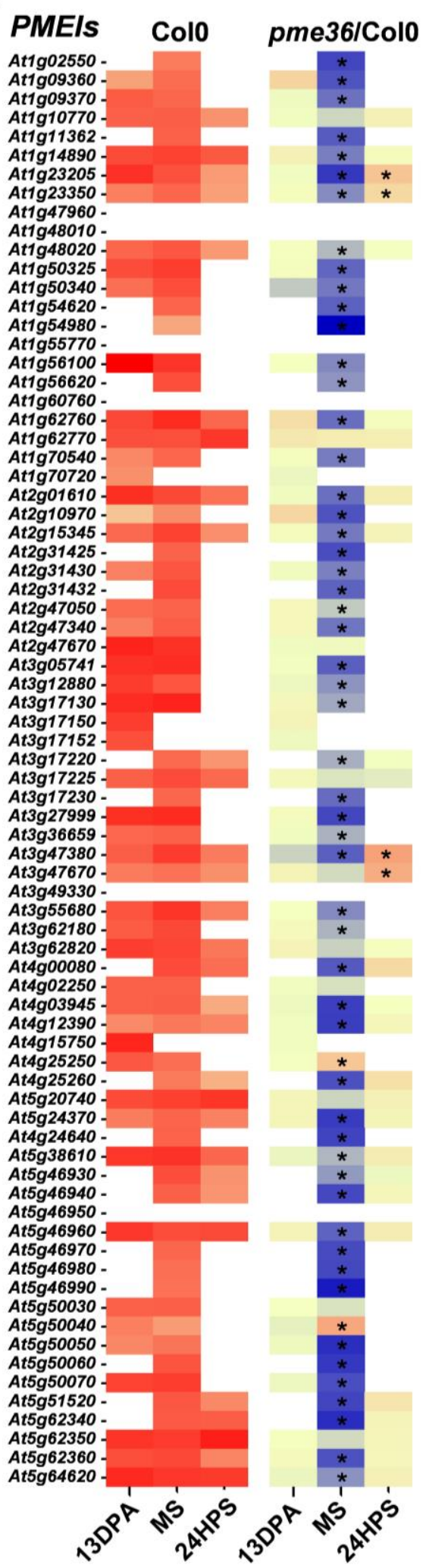

B

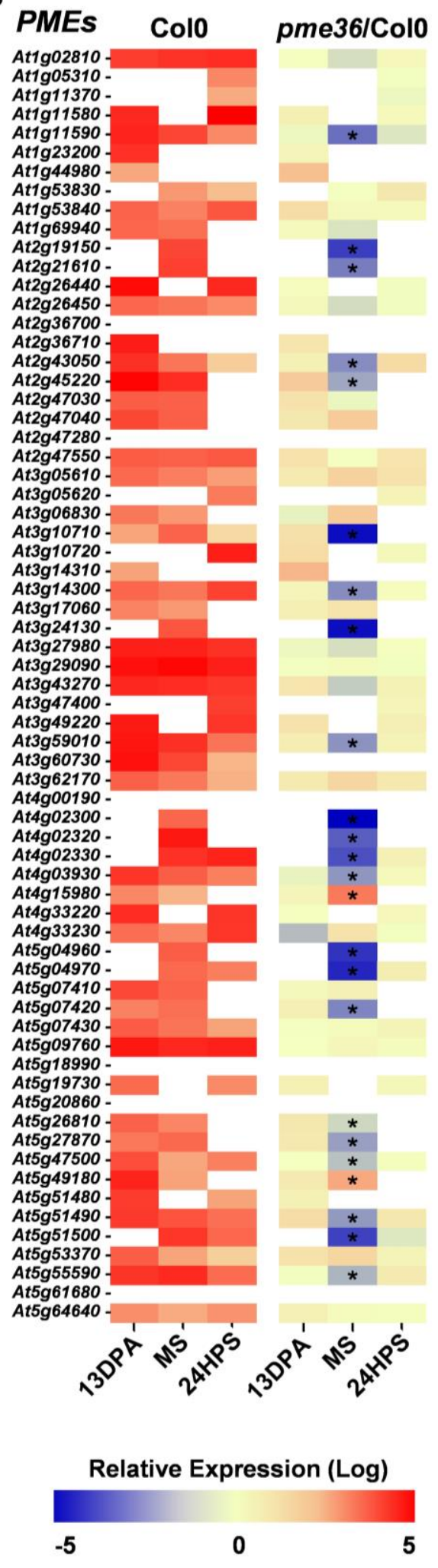

Figure 2. Levels of $P M E$ and PMEI transcripts are strongly downregulated in pme36-1 mature seed.

Quantification by RT-qPCR of PMEI (A) and PME (B) transcripts in 13 days post anthesis (DPA) siliques, in mature seed (MS) and in 24 hours post stratification (HPS) seedlings from Col-0 and pme36-1. Data in Col-0 are normalized non-calibrated gene expression levels (i.e. relative to the reference gene) and data in pme36 are normalized 
data calibrated to the expression levels in the wild type which are set to 1 (i.e. pme361/Col-0). Transcript levels are shown using a logarithmic bicolor scale, in red for upregulation and in blue for downregulation. Empty cells indicate absence of quantifiable transcripts. Values are means from three independent biological replicates. A one-way analysis of variance combined with the Dunnett's comparison posttest confirmed that the differences between the wild type and the mutants $\left(^{*}\right)$ are significant $(P<0.001, n=3)$. 
A

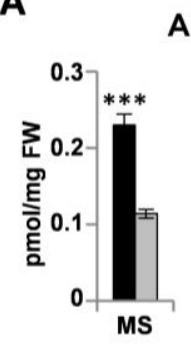

Col0 pme36

C

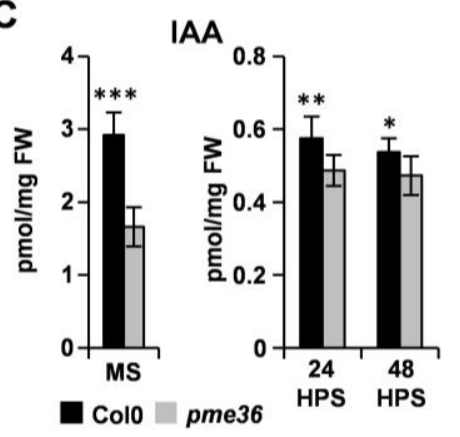

B

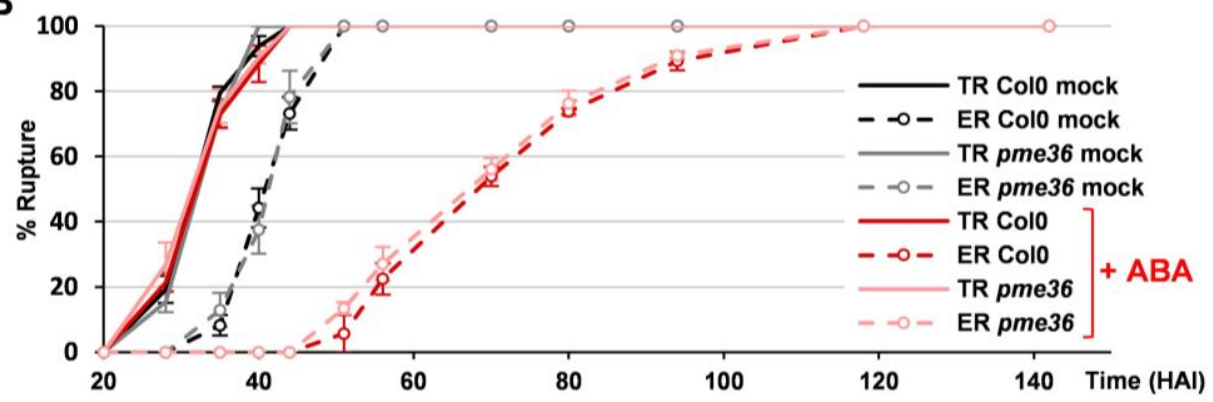

E

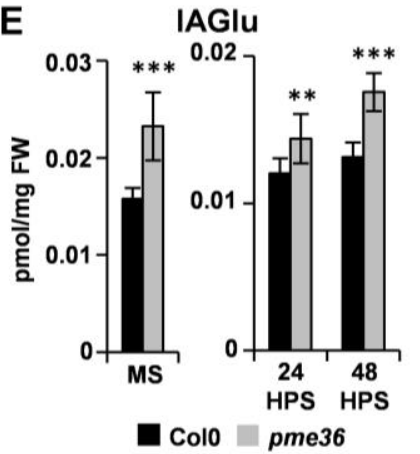

H

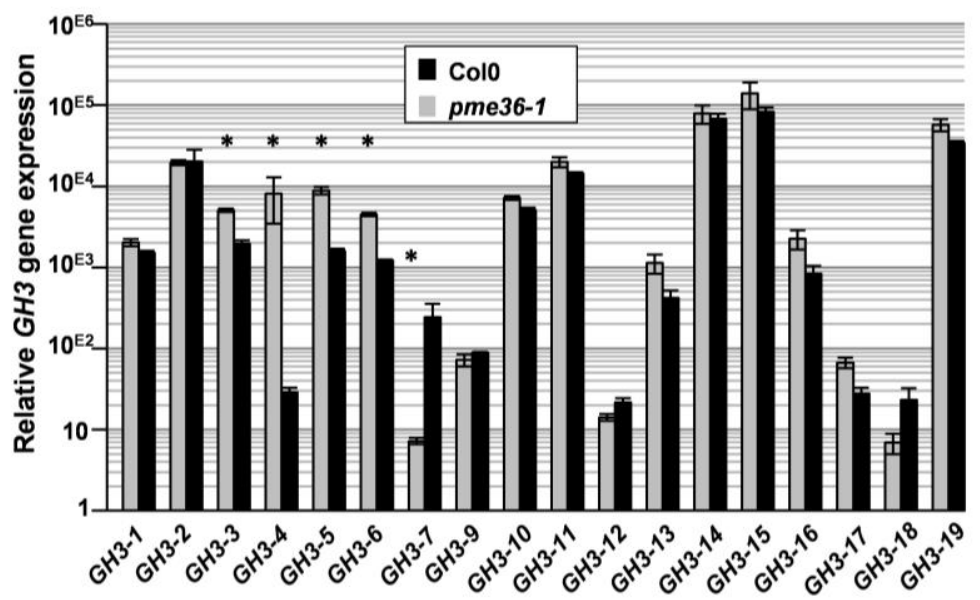

F IAAsp G

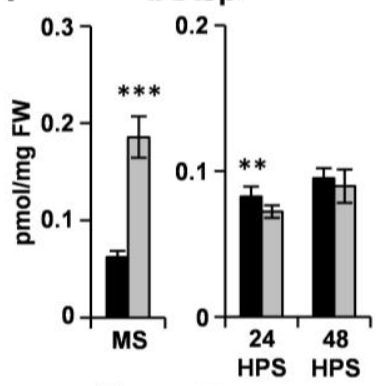

a col0 $\square$ pme36

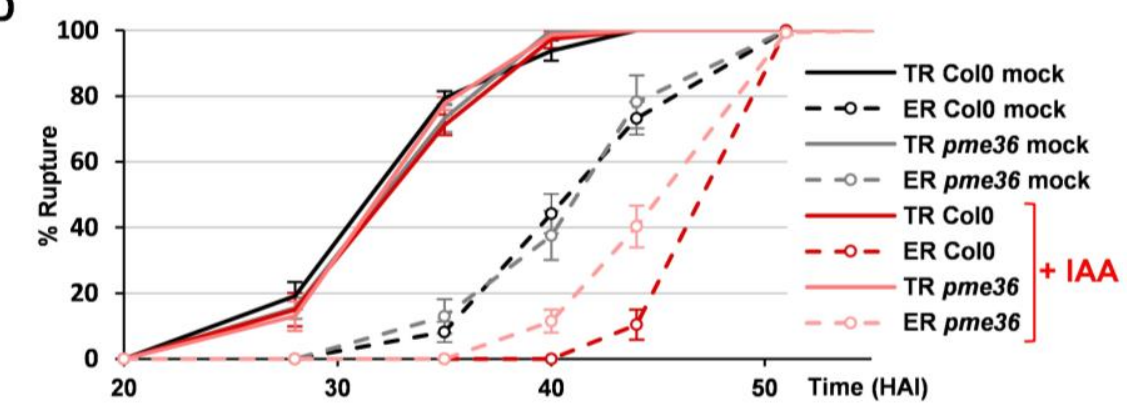

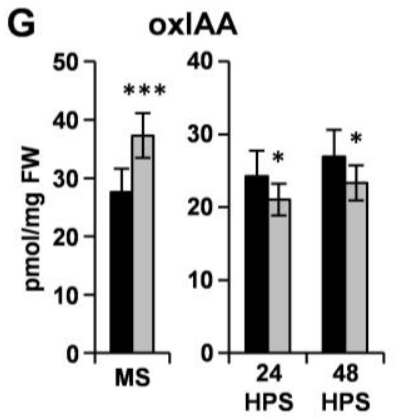

a Col0 pme36
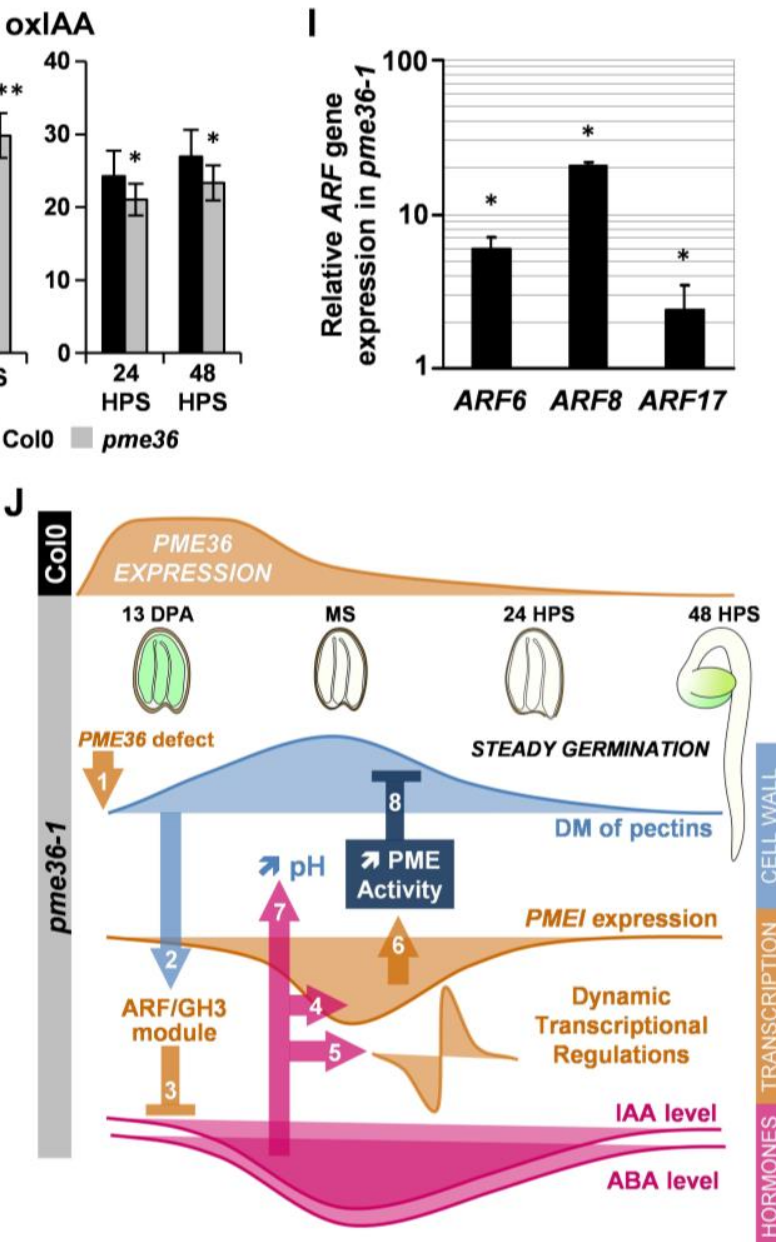

Figure 3. ABA and IAA homeostasis was strongly impaired although hormone sensitivity was not altered in pme36-1 seed.

(A) Quantification of ABA in pme36-1 and Col-0 mature seed (MS) and in 24 and 48 HPS seedlings. Error bars indicate +/-SD of nine biological replicates. ANOVA 
comparison posttest indicated that the values indicated by asterisks were significantly different between wild-type and mutant: $\left({ }^{*}\right)$ for $\mathrm{P}<0.05 ;\left({ }^{* *}\right)$ for $\mathrm{P}<0.01 ;\left({ }^{* * *}\right)$ for $\mathrm{P}<0.001 ; \mathrm{n}=9$. FW: fresh weight. (B) Effect of ABA treatment on germination kinetic of pme36-1 and Col-0 seeds, characterized by testa rupture (TR) and endosperm rupture (ER). Data represent averages and error bars indicate +/- SE, obtained from three independent biological replicates of at least 50 seeds each. (C) Quantification of free IAA in the same conditions as in (A). (D) Effect of IAA treatment on germination kinetic of pme36-1 and Col-0 seeds, analyzed as in (B). (E) to (G) Quantification of IAAsp (N(Indole-3-ylAcetyl)-Aspartate) and IAGlu (N-(Indole-3-ylAcetyl)-Glutamate) conjugates, and oxIAA catabolite, in the same conditions as in $(A)$. $(H)$ Quantification of GH3 transcripts by qRT-PCR in pme36-1 and Col0 mature seed. Gene expression values shown are relative to the expression of TIP41, which has been validated as reference gene in our experimental conditions (see Methods). Error bars indicate +/SE obtained from three independent qPCR experiments. The analysis was performed in two additional independent biological replicates, which gave similar results. A oneway analysis of variance combined with the Tukey's multiple comparison posttest confirmed that the differences between the wild type and the mutants are significant; (*) $\mathrm{P}<0.05, \mathrm{n}=3$. No transcript was detected for $\mathrm{GH} 3-8$ and GH3-20. (I) Quantification of ARF6, ARF8 and ARF17 transcripts by qRT-PCR in pme36-1 and Col-0 13 DPA siliques, analyzed as in $(\mathrm{H})$. (J) Model of combined regulations occurring in pme36-1 seed. 
A

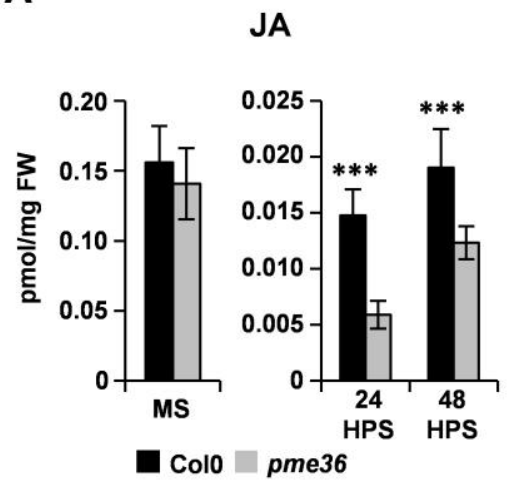

B

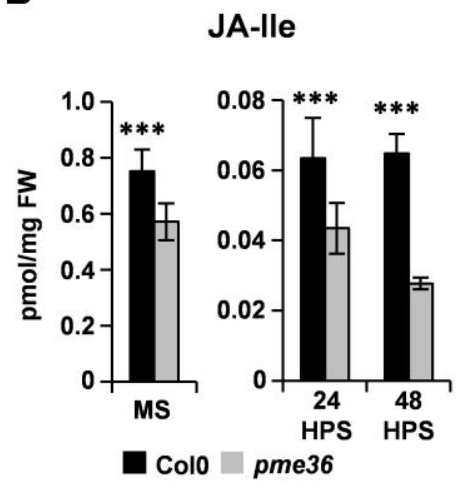

C

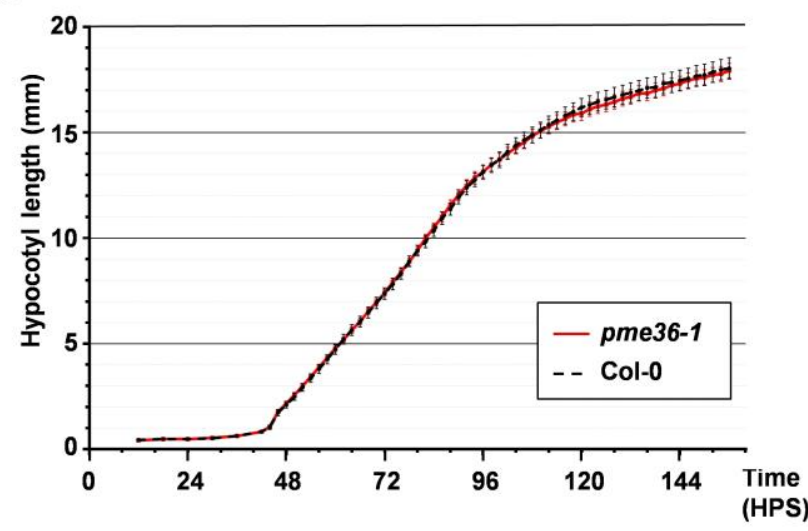

D

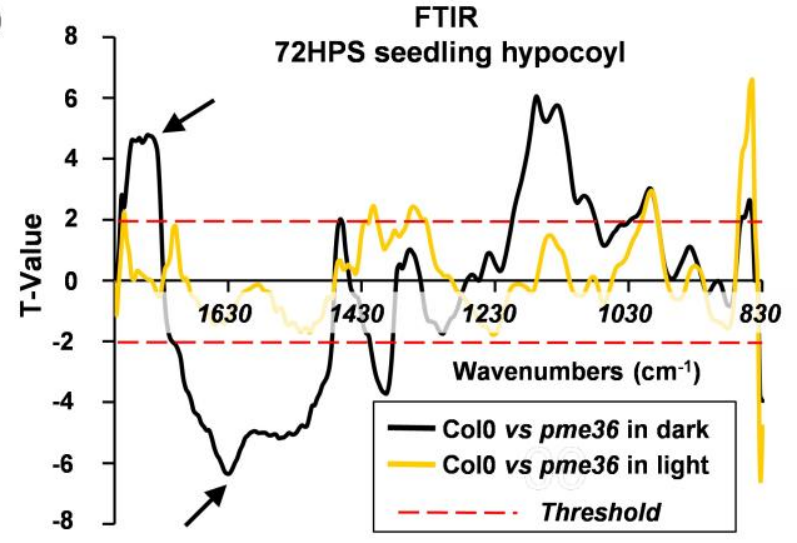

E
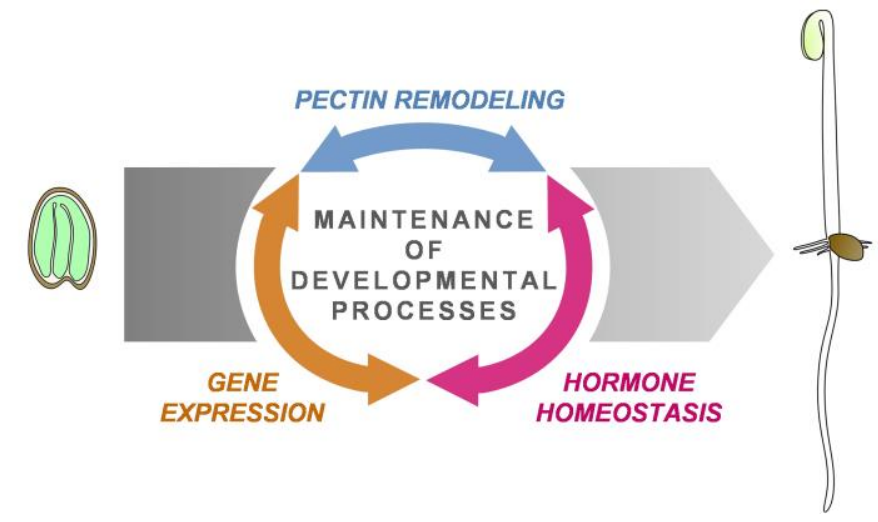

Figure 4. Hypocotyl elongation was not affected by disruption in jasmonate homeostasis and decreased pectin methylesterification in dark-grown pme36-1 seedling.

(A) and (B) Quantification of free JA and JA-lle, respectively, in pme36-1 and Col-0 mature seeds (MS) and in 24 and 48 HPS seedlings. Error bars indicate +/-SD of nine biological replicates. ANOVA comparison posttest indicated that values were significantly different between mutant and wild-type ; $\left(^{* *}\right) \mathrm{P}<0.001, \mathrm{n}=9$. FW: fresh weight. (C) Measurement of hypocotyl length in Col-0 and pme36-1 seedlings grown in the dark for 6 days. Seedlings were imaged with RGB camera every 2 hours. Errors bars indicate +/-SE obtained from 90 seedlings (i.e. one biological replicate) for each line. The graph shows data from one of the two biological replicates. An ANOVA comparison indicated that the values were not significantly between the 2 lines $(p<0,05, n=90)$. (D) Student t-test on the comparison between Fourier TransformInfrared (FT-IR) spectra collected on hypocotyls from Col0 and pme36-1 72 HPS seedlings grown in the dark or in long-day light condition. Arrows indicate wavenumbers $1785 \mathrm{~cm}^{-1}$ and $1630 \mathrm{~cm}^{-1}$, for COOR linkages and COO- linkages, respectively, corresponding to pectins for which the differences are significant between the mutant and the wild type $(P<0,01)$. (E) Model of the homeostatic system highlighted in this study. 


\section{SUPPLEMENTAL MATERIALS}

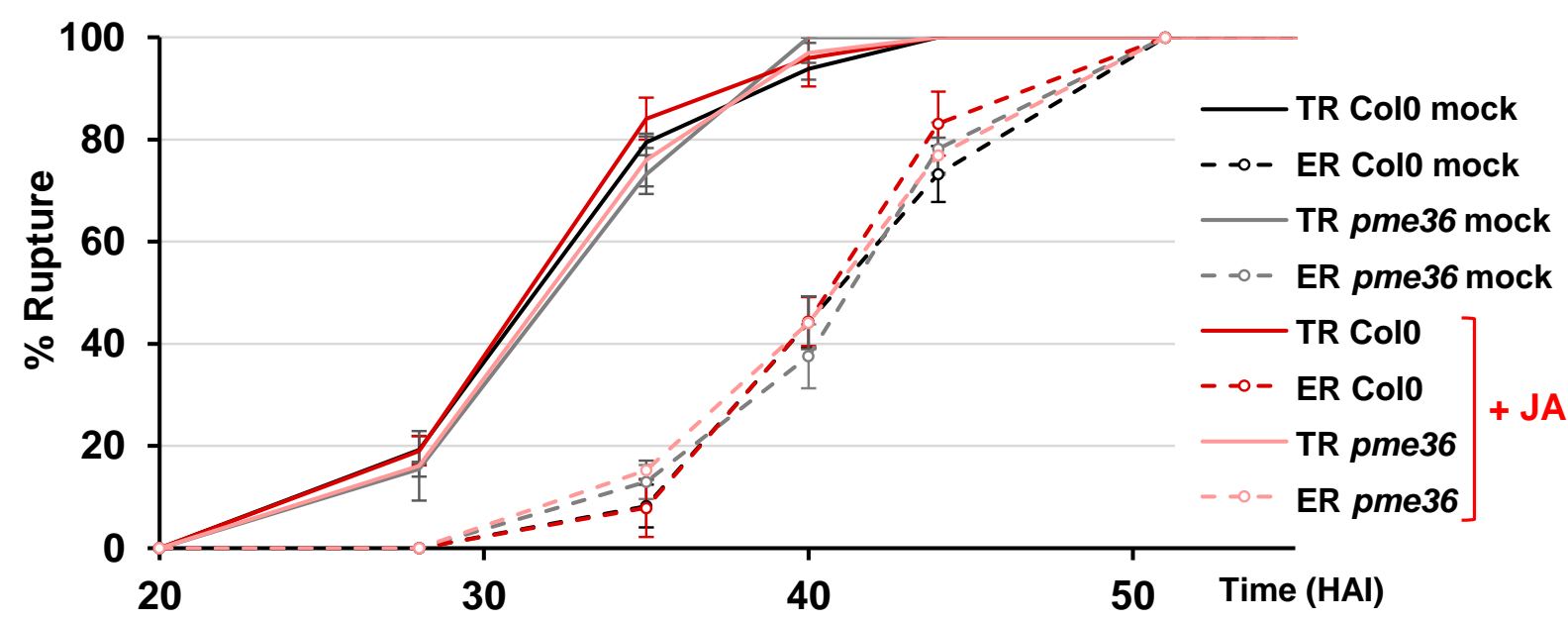

Supplemental Figure 1. JA treatment has no effect on Col-0 and pme36-1 germination.

Effect of JA treatment on germination kinetic of pme36-1 and Col-0 seeds, characterized by testa rupture (TR) and endosperm rupture (ER). Data represent averages and error bars indicate +/- SE, obtained from three independent biological replicates of at least 50 seeds each.

Supplemental Dataset 1. Genes differentially expressed in 16 and 24 HPS pme361 seedlings compared to Col-0.

Supplemental Video 1. Kinetic of seed germination and hypocotyl elongation in pme36-1 and Col-0 seedlings grown in the dark.

Supplemental Video 2. Effect of JA treatment on the kinetic of seed germination and hypocotyl elongation in pme36-1 and Col-0 seedlings grown in the dark. 
bioRxiv preprint doi: https://doi.org/10.1101/2021.07.22.453319; this version posted July 23,2021 . The copyright holder for this preprint

(which was not certified by peer review) is the author/funder, who has granted bioRxiv a license to display the preprint in perpetuity. It is made available under aCC-BY-NC-ND 4.0 International license.

\begin{tabular}{|c|c|c|}
\hline \multicolumn{3}{|c|}{ PMEI } \\
\hline Accession & Forward Primer & Reverse Primer \\
\hline At1g02550 & ATCTCATCCCTTCACGTGTCAC & TCGAATTTGCGGAAGTCACG \\
\hline At1g09360 & ACGAGTGGTCTCAAGACTTGTC & TTGGCTGTGGACAAAATGGC \\
\hline At1g09370 & TCAACGGAATCTGCAAGCAG & AATAAGTGTTGGCCGCGAAC \\
\hline At1g10770 & AGCACGTGTTACGAGACATTGGG & TATGCTCTITCTCGCGCTTGCC \\
\hline At1g11362 & ACAGATGTCGATGCTTCATTGTGC & CCAGTGCTGCAATTTGAGGAGATG \\
\hline At1g14890 & AATACACGGCGGCTTACCTCTC & TTCGAAACGCAGTCGTGGACAG \\
\hline At1g23205 & TGGCTCAGTGCGGCTTAACTG & TCCTCGGCTCATCGTGAATGTC \\
\hline At1g23350 & TTGATGAAGCGGACGAGGCAAG & TCCATCGCGTTTCAGCTCTTC \\
\hline At1g47960 & GGGAGTTCTCCGTTGACTAGTTTG & AGAAGGCCACGAAGATCCACAAG \\
\hline At1g48010 & AAGCGAGCACGGTAGTGAGAAG & TGGCAACGCCAGGAAGATCAAG \\
\hline At1g48020 & TCGCATCGCCTAATCTTCAAGCC & ACGTTTGTGTAGCTCTCGCTTGTG \\
\hline At1g50325 & CCGCACTTAAAGCTTTGGCAACC & TTCCATCGCGAATTGAGACATAGC \\
\hline At1g50340 & CTCACAAACAACACAACAGATCGC & AGCGGTTCAAGCGGATCAATAAG \\
\hline At1g54620 & AGGGCAGGCTGAGTAAGAAGTTG & AGGTTAACGCGTCATAGCCTTCAG \\
\hline At1g54980 & ACAGTTCAAGGAGTGCCAAG & СTCСTTCACTCGCTTTTTTCG \\
\hline At1g55770 & TCTCCTTCGTCACCGGAAATGC & TGGATCGTCGGAGTGTTGCAG \\
\hline At1g56100 & AGACCGTGTTG & \\
\hline At1g56620 & TACAGAGC & AATAATCACCT \\
\hline At1g60760 & AAGC & TCGACACAAGC \\
\hline At1g62760 & TCTCCTТACGCСТCAACAATCCG & TGAGATTGAGGGCGATGACAGC \\
\hline At1g62770 & CGTCGCCAAACTAACCAAAGAGAO & AGCTAACCGGTCCACGCTATTG \\
\hline At1g70540 & AGTGCCTCCTTGACGGACTTG & ACATTCGAAGCCGTACCACCAC \\
\hline At1g70720 & GATTC & GAGTG \\
\hline At2g01610 & CGG & GAG \\
\hline & TGG & TGG \\
\hline At2g15345 & AAGCAGTTCTGTCTCGCTTGGC & TTGCATGTCAAGGGTGAACGTG \\
\hline At2g31425 & ATAACCCGAAACACGCAAGCTG & TTGCCCATTCGTCTTCGCACTC \\
\hline & AAACGCGATTCCAGCCAGAGAC & CAGTTG \\
\hline At2g31432 & TCTG & GCG \\
\hline At2g47050 & AAACC & CTTC \\
\hline At2g47340 & ATAGG & \\
\hline At2g47670 & ATACAAGTCGCTCGCCGTTCTG & ATCGCTTATCTGCCGCTGGAAC \\
\hline At3g05741 & ACGCAAGCACTTGTGGCTATTTC & AGGCCCTTCGAACGCATCTTTG \\
\hline At3g12 & ACAATCCCACCTTGTGTCTTCGC & \\
\hline At3g17130 & TTCTGC & ATAGGTCGCA \\
\hline At3g17150 & ATCC & AAGCTCTC \\
\hline & ACTC & ACCTTGTAG \\
\hline At3g17220 & TCAGGTGCTGATCTTCAAACGC & ACCTTCTGATGCACTTGTTTGTGC \\
\hline At3g17225 & GCACAGAACCGCACATTGACAC & TCGCCGCTAGGTTCTTATTGCC \\
\hline At3g17230 & $\overline{A C G C}$ & \\
\hline At3g27999 & GCTACATGCC & CCAC \\
\hline At3g36659 & AAAGTCGACGCCTCCGATTGTC & TGACGGCGTTAACTGCATCTTCC \\
\hline At3g47380 & ACCCAACCGTATGCGTCAACTC & ACGTCGAGGGCTTGTTGGATG \\
\hline At3g47670 & TTAACCGCCGGAGCTTTCACTC & ACTTGAGAGAGGCGGAGGAGATAG \\
\hline At3g49330 & TCACATCTCAGCTAGTCAGACGAC & GCTGCGGTTAGTTCGCGATTG \\
\hline At3g5 & $\overline{A G C A}$ & ACTCGAGCC \\
\hline At3g62180 & AAACACATGT & GCGAAATCTTCCAACGCC \\
\hline At3g62820 & TACACGCTACCAAGACCTC & \\
\hline At4g00080 & TGCACTCATGGTGCCGAACAAC & ACGCACGTAACGAGACATCGAC \\
\hline At4g02250 & CGACGTCGATATTTGGCTCAACAC & TACCGGCACGATCGTTCAAAGC \\
\hline At4g03945 & TTCGACCGGCGTGATGAAG & TCCACCGACCATAGTGTTTACG \\
\hline At4g12390 & CATC & GTAACGC \\
\hline At4g15750 & CAATATCGACCGTGCTGG & ACTITAGACTCGTGACGGTGTTG \\
\hline At4g24640 & & AAGTAGGCCTTGTGGCACTCTG \\
\hline At4g25250 & AAACGGCATGCAACTCAACAAC & CGGACTTGATGGTGGAGGAATAGG \\
\hline At4g25260 & AGCATAGCCCGAGCAAAGTCAG & TCGTGCTGCCTCTTTGTGATTCC \\
\hline At5g20740 & AAACGGAGGTGAAGCAGTGGATG & GACCATGTAAAGCGCGTTGCTC \\
\hline At5g24370 & ACTCGCCGCACGCAAATATAGC & AACCGTCTCCGTAGCAAACGTC \\
\hline At5g38610 & CGCAACAGCTTCCACCATTCAAC & AGGCAAGCACTGCGGAAACTAC \\
\hline At5g46930 & TGGAGAGGCCGTTACTTGATTGC & AACTTCCCGGTGCATCCATTGC \\
\hline At5g46940 & TCGCCGGATTAGTCATGGCATC & TCCTITCAAGGTCGTCGCTTIGG \\
\hline At5g46950 & ATCCACAAAGCAAAGCCGCAAC & GCAACGTTCTITGTCGATGCTAGG \\
\hline At5g46960 & TGAGTGCTGCTTGGGATGTACCAG & TGTTCTCGTTGGTAATCGGAGACG \\
\hline At5g46970 & ACAACTTCTGTGTCAAGTCTCTCG & CAACCTGTCAAGACTACGTGCAG \\
\hline At5g46980 & TGGCCAATAGAGTGGCGGATTC & GGCTCTCTGGATTCTCTTGATGG \\
\hline At5g46990 & CGTCATGCAAGAGTTGTTCC & CATCGTTCTCTTTCGTCACC \\
\hline At5g50030 & TGGCGATGATACAAGGCGTGTG & AGCGTCTTGGAAGCAGTGTCAG \\
\hline At5g50040 & TTCGGCCACAACTTACATTGACG & TGGCCGTAGCTGCTTGAAACTG \\
\hline At5g50050 & GCTTCGGAGGAACTCTATGATG & TCATCGTCGCTATGTTGTCG \\
\hline At5g50060 & TCAACCTCGGCATATCCTACGC & TTGGCTCTTCTTAGCCGCTTCG \\
\hline At5g50070 & AAGGAGCCGAGCTTGAAGACAC & TGCGATATGTGGATCGCGCTTG \\
\hline At5g51520 & ACGTGAAATCCGCAAAGGAAGC & TTCGTCCGGCGGTTGATTCTG \\
\hline At5g62340 & TGGACCGACTGCTTGTTCACG & GCTATGACTCTCACCCGAAATGGC \\
\hline At5g62350 & ACGATACCGTTGACCGTTIGACC & TGGCACTCCCACACAACTTCAG \\
\hline At5g62360 & TGCGGCTCTCAACATCACATTAGC & TCGAGAGACGCACCATCATTGC \\
\hline At5g64620 & TGGCTTATCCGGTGGAGATTCG & AGAATCCCGGAGACAACACCAC \\
\hline
\end{tabular}

\begin{tabular}{|c|c|c|}
\hline \multicolumn{3}{|c|}{ PME } \\
\hline Accession & Forward Primer & Reverse Primer \\
\hline At1g02810 & AGACGGTGGTCACCGGAAATAG & TCACAGCAAATGTGGCGGAATTG \\
\hline At1g05310 & TGAACGTGGCACCGATACCAAAG & ПTСTTATTGCCACCGCCTGAGC \\
\hline At1g11370 & AAACCGGGGATAGGACAAAC & CTGGAACGAAAATCCCGAAG \\
\hline At1g11580 & TTCCAATCGCTTCCCGTCGTG & TCGCGGTCACCTTAAGTGTCTTG \\
\hline At1g11590 & CGCTCAGTCACGCGCATTAAG & TCCGAGCTTGCAGTAATGTTGC \\
\hline At1g23200 & ACATCTACGCTCGTAAGCCGATG & TTGCGTGATTGAGCGGTGATCG \\
\hline At1g44980 & AGAACGTTCAACACCGCAAC & TTCACACGGAGGGTAACAAC \\
\hline At1g53830 & TACGACGACGCCGATAGGAAAG & ATGTGCTCCACGTGTACCTGAC \\
\hline At1g53840 & ACGGCGACGTTTGCTATACAAGG & TGCTCCCGCGGTGTTATGATTC \\
\hline At1g69940 & CCAGGATCACACAAAGCCAAG & GAGTTTACAAAGCGGGTGGTG \\
\hline At2g19150 & TAACGGCACAAGGAAGGGACTC & TTGCCGCTTCCCATAACAGTGC \\
\hline At2g21610 & AGAAGCTACCGTCTTCTTGAGC & TGGGAGCATTCTTGAAGTGAG \\
\hline At2g26440 & ACTTCCGATCTGCTACTCTTGCG & TAGTGCAACCGCTTGGTGCTTC \\
\hline At2g26450 & GGTCATAAACAAGGAGGAGGCTाT & AGCCTGAGGCACTGATCCAA \\
\hline At2g36700 & GGGAGATCCCTTACGAGGATTGC & TCTTGCACTTCCCGTGATCTTGC \\
\hline At2g36710 & CGGAAACACTGCTGATAGCTTCTC & TCTGGTTCTGGTGCATTGTTCTTG \\
\hline At2g43050 & TCCGCCGTCGTATTCAATCCTG & TTGCGCCGTCACGAAGTTCTC \\
\hline At2g45220 & AGCAAGGCGTTTACAGTGAGAACC & CCGCGACAGTAGCTGAGTTAAAGG \\
\hline At2g47030 & ACAACAGAAGTGTTGCTCTCAGC & AACCTGAACTGTGGCACTGAGG \\
\hline At2g47040 & TTCCCTTAGTGGCACAGTCCAG & CGTTCACTCTGATAGCCACAGC \\
\hline At2g47280 & CGTCGTTCTTCC & AACGTTAAGTC \\
\hline At2g47550 & TGAGTGCTGC & ССТTCTTGGC \\
\hline At3g0 & TTCATCG & AGTAGTTCCAAC \\
\hline At3g05620 & CGGTGACCGGAATTTCATGCAG & ATCCTCTTCCGGAAACAGCAAC \\
\hline At3g06830 & GACCTTCCTAACAGCCACAATCAC & TCCAGCTGTGTTCTCGATTCCG \\
\hline At3g10710 & CGGTTCCTGAGCTAGTCGAT & GCCTCATATCTGGAGCCAAC \\
\hline At3g10720 & GTCCGGCTGTGTTCTG & GCTTCGC \\
\hline At3g1 & GCAAAGG & GGCGGTGTT \\
\hline At $3 g$ & CCGGAACC & AGCG \\
\hline At3g17060 & TCGATGCTGTCCCTGTTGGTAAC & CGGAATGTGCACTCTCTCCTTG \\
\hline At3g24130 & TGCCTCGTATATGGAACAGG & TCCCATCCTTCAGGAACAAC \\
\hline At3g27980 & CGGAGGAGAATTCCCACACT & GGCGTTATAGGCACCTTCA \\
\hline At3g29090 & GGGAAACGC & ACCCGGACCAAAGCACCTATAC \\
\hline At3g43270 & TGCTACATTTGCTGTGAGCGGTAG & TTGGTAACCTCTCA \\
\hline At3g47400 & SGGCTTG & AACA \\
\hline At3g49220 & CTTCCACACGGCGTCATTTGC & TAGATCACTGCGTGGTCAGCTC \\
\hline At3g59010 & CCGCCATGGGAGATGGATTCATAG & AGTTGGTCCGGCACTGTTCAC \\
\hline At3g60730 & AGGCGGCCTATGGATCATCAAG & GCCTGAATTTGTATGTGGGTCGTC \\
\hline At3g62170 & GTTCAG & TGTTC \\
\hline At4g00190 & TCAAAGCCAAGAGTGACACG & AGCAAACGTTCTTGGGACAC \\
\hline At4g02300 & ACCTTCCCCGAAATCATCAG & GTTTCCGGTACCATCAATCG \\
\hline At4g02320 & ACTTCAAGACCCGGTGGATGAG & AACCTGGTTCACTCGAGTTGGG \\
\hline At4g02330 & TACATCGCCGAACTTCGTTGCC & GCTTCTCTGGTCCAGCGGTATTTC \\
\hline At $4 \mathrm{~g} 03930$ & CCTTGGGAAGGAGAAACTGGTCTG & TCACCCTTCTGCTAGTCACAGCTC \\
\hline At4g15980 & TCTTCCCGTGAAAGCCAAGAAC & CGCAAACGTCTCGTTCCACTTC \\
\hline At4g33220 & CACTTTCCGATCAGCTACATTCGC & ATCTCAGTGCTACCGCCTGATG \\
\hline At4g33230 & TGCCAAGTACCAAGGAAGGTACAC & TGTTCCCGTCACGATCGTCTTC \\
\hline At5g04960 & GGCGAACACATGGATAACG & GTACGTTCCCACGGAGCTAA \\
\hline At5g04970 & GGGGGCTGATACAAGTAAAAGG & ACGGTGAAGTTCATGGCTTG \\
\hline At5g07410 & AGACTCCCGCACATGACAAGAC & САCTCTCTTGGCTTGTGTGACC \\
\hline At5g07420 & AAACACTTСТССАATGCСAAAGCC & GAAAGCAGCCTTA \\
\hline At5g07430 & CGAAGTTACGCCTTTCCTCACTC & GAGGAGGAAGTAGCCATGTGGA \\
\hline At5g09760 & GGTTCGTTAAACGCCGGTATGC & TCTCCTACCACTCCGACAGTTG \\
\hline At5g18990 & GAGCGGCCAATCTATTTACCAGAG & ACGAATCCATTCGCGTCGTTTG \\
\hline At5g19730 & TाTGTATCTTGGGAGGGCATGGG & ACTGCCCATAGAACACCGTCATC \\
\hline At5g20860 & TCGTCGATGGGTTCCTCCAAAC & TGAGCCGTTCACAAGAATCTGAG \\
\hline At5g26810 & AGTGGCCAATCTGTTTACGAGGAC & TCGTAGGAACACGAACCCACTTG \\
\hline At5g27870 & GCGAGAATCCACGGGATTTGTTC & ACTGCCAAGTAATCCGGTTCGC \\
\hline At5g47500 & ACCTATCAAACCGCTTCCGTCAC & GGCGCAGTATTCGTGAAGCTAATG \\
\hline At5g49180 & ACATACCTTACTGCCACTGTTGCG & AAATCCGCCGAGACTCTTAGGG \\
\hline At5g51480 & AACTTCCACGTTTGTCCAGCTC & TGACCATATACCAAAGTCCATCGC \\
\hline At5g51490 & GGTCGGAGATGGAATGAGATCCAC & GATACCGGCAGTAGCGGAATTG \\
\hline At5g51500 & AACACAGCGGTTTCGACTG & TGGTCAATTTGTCCCAAGCG \\
\hline At5g53370 & TTCACACCGCCACTTCGC & TTCTCAAACGTCATGTCCCTCAC \\
\hline At5g55590 & TTTACAGGGAGAAGGTGATTGTGC & TCTCCCGCATAGCTCTCATTACC \\
\hline At5g61680 & AGCAGTGGTTCAACACCAACGTG & TGATCACACGCCTAGTGTTCCC \\
\hline At5g64640 & ACAGGGTCGTTGAATGTTGGAC & TCACCGAGAACTCCGACAGTTG \\
\hline
\end{tabular}

Supplemental Table 1. Sequences of primers designed for RT-qPCR on PME and PMEl genes. 